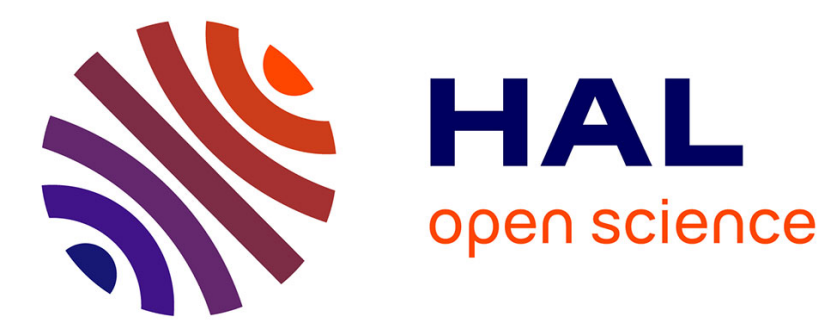

\title{
Jets and energy flow in photon-proton collisions at HERA
}

\author{
S. Aid, V. Andreev, B. Andrieu, R D. Appuhn, M. Arpagaus, A. Babaev, J. \\ Bhr, J. Bn, Y. Ban, P. Baranov, et al.
}

\section{> To cite this version:}

S. Aid, V. Andreev, B. Andrieu, R D. Appuhn, M. Arpagaus, et al.. Jets and energy flow in photonproton collisions at HERA. Zeitschrift für Physik C Particles and Fields, 1995, 70, pp.17-30. in2p300013130

\section{HAL Id: in2p3-00013130 https://hal.in2p3.fr/in2p3-00013130}

Submitted on 23 Mar 1999

HAL is a multi-disciplinary open access archive for the deposit and dissemination of scientific research documents, whether they are published or not. The documents may come from teaching and research institutions in France or abroad, or from public or private research centers.
L'archive ouverte pluridisciplinaire HAL, est destinée au dépôt et à la diffusion de documents scientifiques de niveau recherche, publiés ou non, émanant des établissements d'enseignement et de recherche français ou étrangers, des laboratoires publics ou privés. 


\title{
Jets and Energy Flow in Photon-Proton Collisions at HERA
}

\author{
H1 Collaboration
}

\begin{abstract}
:
Properties of the hadronic final state in photoproduction events with large transverse energy are studied at the electron-proton collider HERA. Distributions of the transverse energy, jets and underlying event energy are compared to $\bar{p} p$ data and QCD calculations. The comparisons show that the $\gamma p$ events can be consistently described by QCD models including - in addition to the primary hard scattering process - interactions between the two beam remnants. The differential jet cross sections $d \sigma / d E_{T}^{j e t}$ and $d \sigma / d \eta^{j e t}$ are measured.
\end{abstract}




\section{H1 Collaboration}

S. Aid ${ }^{14}$, V. Andreev ${ }^{26}$, B. Andrieu ${ }^{29}$, R.-D. Appuhn ${ }^{12}$, M. Arpagaus ${ }^{37}$, A. Babaev ${ }^{25}$, J. Bähr ${ }^{36}$, J. Bán ${ }^{18}$, Y. Ban ${ }^{28}$, P. Baranov ${ }^{26}$, E. Barrelet ${ }^{30}$, R. Barschke ${ }^{12}$, W. Bartel ${ }^{12}$, M. Barth ${ }^{5}$, U. Bassler ${ }^{30}$, H.P. Beck ${ }^{38}$, H.-J. Behrend ${ }^{12}$, A. Belousov ${ }^{26}$, Ch. Berger ${ }^{1}$, G. Bernardi ${ }^{30}$, R. Bernet ${ }^{37}$, G. Bertrand-Coremans ${ }^{5}$, M. Besançon ${ }^{10}$, R. Beyer ${ }^{12}$, P. Biddulph ${ }^{23}$, P. Bispham ${ }^{23}$, J.C. Bizot ${ }^{28}$, V. Blobel ${ }^{14}$, K. Borras ${ }^{9}$, F. Botterweck ${ }^{5}$, V. Boudry ${ }^{29}$, S. Bourov ${ }^{25}$, A. Braemer ${ }^{15}$, F. Brasse ${ }^{12}$, W. Braunschweig ${ }^{1}$, V. Brisson ${ }^{28}$, D. Bruncko ${ }^{18}$, C. Brune ${ }^{16}$, R. Buchholz ${ }^{12}$, L. Büngener ${ }^{14}$, J. Bürger ${ }^{12}$, F.W. Büsser ${ }^{14}$, A. Buniatian ${ }^{12,39}$, S. Burke ${ }^{19}$, M.J. Burton ${ }^{23}$, G. Buschhorn ${ }^{27}$, A.J. Campbell ${ }^{12}$, T. Carli ${ }^{27}$, F. Charles ${ }^{12}$, M. Charlet ${ }^{12}$, D. Clarke ${ }^{6}$, A.B. Clegg ${ }^{19}$, B. Clerbaux ${ }^{5}$, J.G. Contreras ${ }^{9}$, C. Cormack ${ }^{20}$, J.A. Coughlan ${ }^{6}$, A. Courau ${ }^{28}$, Ch. Coutures ${ }^{10}$, G. Cozzika ${ }^{10}$, L. Criegee ${ }^{12}$, D.G. Cussans ${ }^{6}$, J. Cvach ${ }^{31}$, S. Dagoret ${ }^{30}$, J.B. Dainton ${ }^{20}$, W.D. Dau ${ }^{17}$, K. Daum ${ }^{35}$, M. David ${ }^{10}$, C.L. Davis ${ }^{19}$, B. Delcourt ${ }^{28}$, L. Del Buono ${ }^{30}$, A. De Roeck ${ }^{12}$, E.A. De Wolf ${ }^{5}$, P. Dixon ${ }^{19}$, P. Di Nezza ${ }^{33}$, C. Dollfus ${ }^{38}$, J.D. Dowell ${ }^{4}$, H.B. Dreis ${ }^{2}$, A. Droutskoi ${ }^{25}$, J. Duboc ${ }^{30}$, D. Düllmann ${ }^{14}$, O. Dünger ${ }^{14}$, H. Duhm ${ }^{13}$, J. Ebert ${ }^{35}$, T.R. Ebert ${ }^{20}$, G. Eckerlin ${ }^{12}$, V. Efremenko ${ }^{25}$, S. Egli ${ }^{38}$, H. Ehrlichmann ${ }^{36}$, S. Eichenberger ${ }^{38}$, R. Eichler ${ }^{37}$, F. Eisele ${ }^{15}$, E. Eisenhandler ${ }^{21}$, R.J. Ellison ${ }^{23}$, E. Elsen ${ }^{12}$, M. Erdmann ${ }^{15}$, W. Erdmann ${ }^{37}$, E. Evrard ${ }^{5}$, L. Favart ${ }^{5}$, A. Fedotov ${ }^{25}$, D. Feeken ${ }^{14}$, R. Felst ${ }^{12}$, J. Feltesse ${ }^{10}$, J. Ferencei ${ }^{16}$, F. Ferrarotto ${ }^{33}$, K. Flamm ${ }^{12}$, M. Fleischer ${ }^{9}$, M. Flieser ${ }^{27}$, G. Flügge ${ }^{2}$, A. Fomenko ${ }^{26}$, B. Fominykh ${ }^{25}$, M. Forbush ${ }^{8}$, J. Formánek ${ }^{32}$, J.M. Foster ${ }^{23}$, G. Franke ${ }^{12}$, E. Fretwurst ${ }^{13}$, E. Gabathuler ${ }^{20}$, K. Gabathuler ${ }^{34}$, J. Garvey ${ }^{4}$, J. Gayler ${ }^{12}$, M. Gebauer ${ }^{9}$, A. Gellrich ${ }^{12}$, H. Genzel ${ }^{1}$, R. Gerhards ${ }^{12}$, A. Glazov ${ }^{36}$, U. Goerlach ${ }^{12}$, L. Goerlich ${ }^{7}$, N. Gogitidze ${ }^{26}$, M. Goldberg ${ }^{30}$, D. Goldner ${ }^{9}$, B. Gonzalez-Pineiro ${ }^{30}$, I. Gorelov ${ }^{25}$, P. Goritchev ${ }^{25}$, C. Grab ${ }^{37}$, H. Grässler ${ }^{2}$, R. Grässler ${ }^{2}$, T. Greenshaw ${ }^{20}$, R.K. Griffiths ${ }^{21}$, G. Grindhammer ${ }^{27}$, A. Gruber ${ }^{27}$, C. Gruber ${ }^{17}$, J. Haack ${ }^{36}$, D. Haidt ${ }^{12}$, L. Hajduk ${ }^{7}$, O. Hamon ${ }^{30}$, M. Hampel ${ }^{1}$, M. Hapke ${ }^{12}$, W.J. Haynes ${ }^{6}$, G. Heinzelmann ${ }^{14}$, R.C.W. Henderson ${ }^{19}$, H. Henschel ${ }^{36}$, I. Herynek ${ }^{31}$, M.F. Hess ${ }^{27}$, W. Hildesheim ${ }^{12}$, P. Hill ${ }^{6}$, K.H. Hiller ${ }^{36}$, C.D. Hilton ${ }^{23}$, J. Hladký ${ }^{31}$, K.C. Hoeger ${ }^{23}$, M. Höppner ${ }^{9}$, R. Horisberger ${ }^{34}$, V.L. Hudgson ${ }^{4}$, Ph. Huet ${ }^{5}$, M. Hütte ${ }^{9}$, H. Hufnagel $^{15}$, M. Ibbotson ${ }^{23}$, H. Itterbeck ${ }^{1}$, M.-A. Jabiol ${ }^{10}$, A. Jacholkowska ${ }^{28}$, C. Jacobsson ${ }^{22}$, M. Jaffre ${ }^{28}$, J. Janoth ${ }^{16}$, T. Jansen ${ }^{12}$, L. Jönsson ${ }^{22}$, K. Johannsen ${ }^{14}$, D.P. Johnson ${ }^{5}$, L. Johnson ${ }^{19}$, H. Jung ${ }^{10}$, P.I.P. Kalmus ${ }^{21}$, D. Kant ${ }^{21}$, R. Kaschowitz ${ }^{2}$, P. Kasselmann ${ }^{13}$, U. Kathage ${ }^{17}$, J. Katzy ${ }^{15}$, H.H. Kaufmann ${ }^{36}$, S. Kazarian ${ }^{12}$, I.R. Kenyon ${ }^{4}$, S. Kermiche ${ }^{24}$, C. Keuker ${ }^{1}$, C. Kiesling ${ }^{27}$, M. Klein ${ }^{36}$, C. Kleinwort ${ }^{14}$, G. Knies ${ }^{12}$, W. Ko ${ }^{8}$, T. Köhler ${ }^{1}$, J.H. Köhne ${ }^{27}$, H. Kolanoski ${ }^{3}$, F. Kole ${ }^{8}$, S.D. Kolya ${ }^{23}$, V. Korbel ${ }^{12}$, M. Korn ${ }^{9}$, P. Kostka ${ }^{36}$, S.K. Kotelnikov ${ }^{26}$, T. Krämerkämper ${ }^{9}$, M.W. Krasny ${ }^{7,30}$, H. Krehbiel ${ }^{12}$, D. Krücker ${ }^{2}$, U. Krüger ${ }^{12}$, U. Krüner-Marquis ${ }^{12}$, H. Küster ${ }^{2}$, M. Kuhlen ${ }^{27}$, T. Kurča ${ }^{18}$, J. Kurzhöfer ${ }^{9}$, B. Kuznik ${ }^{35}$, D. Lacour ${ }^{30}$, B. Laforge ${ }^{10}$, F. Lamarche ${ }^{29}$, R. Lander ${ }^{8}$, M.P.J. Landon ${ }^{21}$, W. Lange ${ }^{36}$, U. Langenegger ${ }^{37}$, P. Lanius ${ }^{27}$, J.-F. Laporte ${ }^{10}$, A. Lebedev ${ }^{26}$, F. Lehner ${ }^{12}$, C. Leverenz ${ }^{12}$,

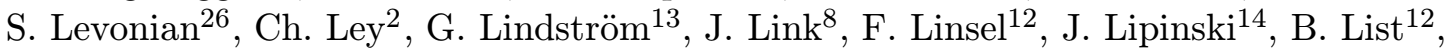
G. Lobo $^{28}$, P. $\operatorname{Loch}^{28}$, H. Lohmander ${ }^{22}$, J.W. Lomas ${ }^{23}$, G.C. Lopez ${ }^{21}$, V. Lubimov ${ }^{25}$, D. Lüke ${ }^{9,12}$, N. Magnussen ${ }^{35}$, E. Malinovski ${ }^{26}$, S. Mani $^{8}$, R. Maraček ${ }^{18}$, P. Marage $^{5}$, J. Marks ${ }^{24}$, R. Marshall ${ }^{23}$, J. Martens ${ }^{35}$, G. Martin ${ }^{14}$, R. Martin ${ }^{20}$, H.-U. Martyn ${ }^{1}$, J. Martyniak ${ }^{28}$, S. Masson ${ }^{2}$, T. Mavroidis ${ }^{21}$, S.J. Maxfield ${ }^{20}$, S.J. McMahon ${ }^{20}$, A. Mehta ${ }^{6}$, K. Meier ${ }^{16}$, D. Mercer ${ }^{23}$, T. Merz ${ }^{12}$, A. Meyer ${ }^{12}$, A. Meyer ${ }^{14}$, C.A. Meyer ${ }^{38}$, H. Meyer ${ }^{35}$, J. Meyer ${ }^{12}$, P.-O. Meyer ${ }^{2}$, A. Migliori ${ }^{29}$, S. Mikocki ${ }^{7}$, D. Milstead ${ }^{20}$, F. Moreau $^{29}$, J.V. Morris ${ }^{6}$, E. Mroczko ${ }^{7}$, G. Müller ${ }^{12}$, K. Müller ${ }^{12}$, P. Murín ${ }^{18}$, V. Nagovizin ${ }^{25}$, R. Nahnhauer ${ }^{36}$, B. Naroska ${ }^{14}$, Th. Naumann ${ }^{36}$, P.R. Newman ${ }^{4}$, D. Newton ${ }^{19}$, D. Neyret ${ }^{30}$, H.K. Nguyen ${ }^{30}$, T.C. Nicholls ${ }^{4}$, F. Niebergall ${ }^{14}$, C. Niebuhr ${ }^{12}$, Ch. Niedzballa ${ }^{1}$, R. Nisius ${ }^{1}$, G. Nowak ${ }^{7}$, G.W. Noyes ${ }^{6}$, M. Nyberg-Werther ${ }^{22}$, M. Oakden ${ }^{20}$, H. Oberlack ${ }^{27}$, U. Obrock ${ }^{9}$, J.E. Olsson ${ }^{12}$, D. Ozerov $^{25}$, P. Palmen ${ }^{2}$, E. Panaro ${ }^{12}$, A. Panitch ${ }^{5}$, C. Pascaud ${ }^{28}$, G.D. Patel ${ }^{20}$, H. Pawletta ${ }^{2}$, E. Peppel ${ }^{36}$, E. Perez ${ }^{10}$, J.P. Phillips ${ }^{20}$, Ch. Pichler ${ }^{13}$, A. Pieuchot ${ }^{24}$, D. Pitzl ${ }^{37}$, G. Pope ${ }^{8}$, 
S. Prell ${ }^{12}$, R. Prosi ${ }^{12}$, K. Rabbertz ${ }^{1}$, G. Rädel ${ }^{12}$, F. Raupach ${ }^{1}$, P. Reimer ${ }^{31}$, S. Reinshagen ${ }^{12}$, P. Ribarics ${ }^{27}$, H. Rick ${ }^{9}$, V. Riech ${ }^{13}$, J. Riedlberger ${ }^{37}$, S. Riess ${ }^{14}$, M. Rietz $^{2}$, E. Rizvi ${ }^{21}$, S.M. Robertson ${ }^{4}$, P. Robmann ${ }^{38}$, H.E. Roloff ${ }^{36}$, R. Roosen ${ }^{5}$, K. Rosenbauer ${ }^{1}$, A. Rostovtsev ${ }^{25}$, F. Rouse ${ }^{8}$, C. Royon ${ }^{10}$, K. Rüter ${ }^{27}$, S. Rusakov ${ }^{26}$, K. Rybicki ${ }^{7}$, N. Sahlmann ${ }^{2}$, D.P.C. Sankey ${ }^{6}$, P. Schacht ${ }^{27}$, S. Schiek ${ }^{14}$, S. Schleif ${ }^{16}$, P. Schleper ${ }^{15}$, W. von Schlippe ${ }^{21}$, D. Schmidt ${ }^{35}$, G. Schmidt ${ }^{14}$, A. Schöning ${ }^{12}$, V. Schröder ${ }^{12}$, E. Schuhmann ${ }^{27}$, B. Schwab ${ }^{15}$, C. Schwanenberger ${ }^{15}$, G. Sciacca ${ }^{36}$, F. Sefkow ${ }^{12}$, M. Seidel ${ }^{13}$, R. Sell ${ }^{12}$, A. Semenov ${ }^{25}$, V. Shekelyan ${ }^{12}$, I. Sheviakov ${ }^{26}$, L.N. Shtarkov ${ }^{26}$, G. Siegmon ${ }^{17}$, U. Siewert ${ }^{17}$, Y. Sirois ${ }^{29}$, I.O. Skillicorn ${ }^{11}$, P. Smirnov ${ }^{26}$, J.R. Smith ${ }^{8}$, V. Solochenko ${ }^{25}$, Y. Soloviev ${ }^{26}$, J. Spiekermann ${ }^{9}$, S. Spielman ${ }^{29}$, H. Spitzer ${ }^{14}$, R. Starosta ${ }^{1}$, M. Steenbock ${ }^{14}$, P. Steffen ${ }^{12}$, R. Steinberg ${ }^{2}$, B. Stella ${ }^{33}$, K. Stephens ${ }^{23}$, J. Stier ${ }^{12}$, J. Stiewe ${ }^{16}$, U. Stößlein ${ }^{36}$, K. Stolze ${ }^{36}$, J. Strachota ${ }^{31}$, U. Straumann ${ }^{38}$, W. Struczinski ${ }^{2}$, J.P. Sutton ${ }^{4}$, S. Tapprogge ${ }^{16}$, V. Tchernyshov ${ }^{25}$,

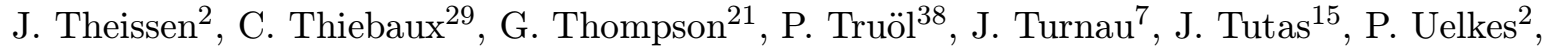
A. Usik ${ }^{26}$, S. Valkár ${ }^{32}$, A. Valkárová ${ }^{32}$, C. Vallée ${ }^{24}$, D. Vandenplas ${ }^{29}$, P. Van $\operatorname{Esch}^{5}$, P. Van Mechelen ${ }^{5}$, A. Vartapetian ${ }^{12,39}$, Y. Vazdik ${ }^{26}$, P. Verrecchia ${ }^{10}$, G. Villet ${ }^{10}$, K. Wacker ${ }^{9}$,

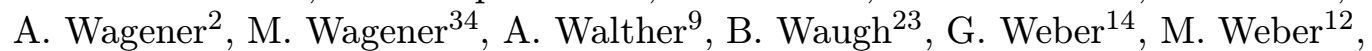

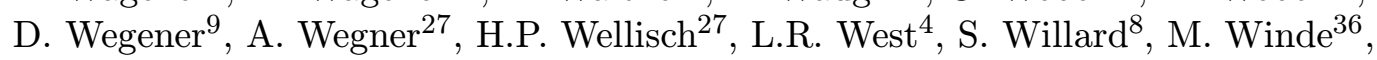
G.-G. Winter ${ }^{12}$, C. Wittek ${ }^{14}$, A.E. Wright ${ }^{23}$, E. Wünsch ${ }^{12}$, N. Wulff ${ }^{12}$, T.P. Yiou ${ }^{30}$, J. Žáček ${ }^{32}$, D. Zarbock ${ }^{13}$, Z. Zhang ${ }^{28}$, A. Zhokin ${ }^{25}$, M. Zimmer ${ }^{12}$, W. Zimmermann ${ }^{12}$, F. Zomer ${ }^{28}$, J. Zsembery ${ }^{10}$, K. Zuber ${ }^{16}$, and M. zurNedden ${ }^{38}$

${ }^{1}$ I. Physikalisches Institut der RWTH, Aachen, Germany ${ }^{a}$

${ }^{2}$ III. Physikalisches Institut der RWTH, Aachen, Germany ${ }^{a}$

${ }^{3}$ Institut für Physik, Humboldt-Universität, Berlin, Germany ${ }^{a}$

${ }^{4}$ School of Physics and Space Research, University of Birmingham, Birmingham, UK ${ }^{b}$

${ }^{5}$ Inter-University Institute for High Energies ULB-VUB, Brussels; Universitaire Instelling Antwerpen, Wilrijk; Belgium ${ }^{c}$

${ }^{6}$ Rutherford Appleton Laboratory, Chilton, Didcot, UK ${ }^{b}$

7 Institute for Nuclear Physics, Cracow, Poland ${ }^{d}$

8 Physics Department and IIRPA, University of California, Davis, California, USA ${ }^{e}$

${ }^{9}$ Institut für Physik, Universität Dortmund, Dortmund, Germany ${ }^{a}$

${ }^{10}$ CEA, DSM/DAPNIA, CE-Saclay, Gif-sur-Yvette, France

11 Department of Physics and Astronomy, University of Glasgow, Glasgow, UK ${ }^{b}$

12 DESY, Hamburg, Germany ${ }^{a}$

13 I. Institut für Experimentalphysik, Universität Hamburg, Hamburg, Germany ${ }^{a}$

14 II. Institut für Experimentalphysik, Universität Hamburg, Hamburg, Germany ${ }^{a}$

15 Physikalisches Institut, Universität Heidelberg, Heidelberg, Germany ${ }^{a}$

16 Institut für Hochenergiephysik, Universität Heidelberg, Heidelberg, Germany ${ }^{a}$

17 Institut für Reine und Angewandte Kernphysik, Universität Kiel, Kiel, Germany ${ }^{a}$

18 Institute of Experimental Physics, Slovak Academy of Sciences, Košice, Slovak Republic ${ }^{f}$

19 School of Physics and Chemistry, University of Lancaster, Lancaster, UK ${ }^{b}$

20 Department of Physics, University of Liverpool, Liverpool, $U K^{b}$

21 Queen Mary and Westfield College, London, UK

22 Physics Department, University of Lund, Lund, Sweden ${ }^{g}$

23 Physics Department, University of Manchester, Manchester, UK ${ }^{b}$

${ }^{24}$ CPPM, Université d'Aix-Marseille II, IN2P3-CNRS, Marseille, France

${ }^{25}$ Institute for Theoretical and Experimental Physics, Moscow, Russia

${ }^{26}$ Lebedev Physical Institute, Moscow, Russia ${ }^{f}$

27 Max-Planck-Institut für Physik, München, Germany ${ }^{a}$

28 LAL, Université de Paris-Sud, IN2P3-CNRS, Orsay, France

${ }^{29}$ LPNHE, Ecole Polytechnique, IN2P3-CNRS, Palaiseau, France

${ }^{30}$ LPNHE, Universités Paris VI and VII, IN2P3-CNRS, Paris, France 
31 Institute of Physics, Czech Academy of Sciences, Praha, Czech Republic ${ }^{f, h}$

32 Nuclear Center, Charles University, Praha, Czech Republic ${ }^{f, h}$

33 INFN Roma and Dipartimento di Fisica, Universita "La Sapienza", Roma, Italy

34 Paul Scherrer Institut, Villigen, Switzerland

35 Fachbereich Physik, Bergische Universität Gesamthochschule Wuppertal, Wuppertal, Germany ${ }^{a}$

${ }^{36}$ DESY, Institut für Hochenergiephysik, Zeuthen, Germany ${ }^{a}$

37 Institut für Teilchenphysik, ETH, Zürich, Switzerland ${ }^{i}$

38 Physik-Institut der Universität Zürich, Zürich, Switzerland

39 Visitor from Yerevan Phys.Inst., Armenia

a Supported by the Bundesministerium für Forschung und Technologie, FRG under contract numbers 6AC17P, 6AC47P, 6DO57I, 6HH17P, 6HH27I, 6HD17I, 6HD27I, 6KI17P, 6MP17I, and $6 \mathrm{WT} 8 \mathrm{r} P$

${ }^{b}$ Supported by the UK Particle Physics and Astronomy Research Council, and formerly by the UK Science and Engineering Research Council

${ }^{c}$ Supported by FNRS-NFWO, IISN-IIKW

${ }^{d}$ Supported by the Polish State Committee for Scientific Research, grant Nos.

SPUB/P3/202/94 and 2 PO3B 237 08, and Stiftung fuer Deutsch-Polnische Zusammenarbeit, project no.506/92

e Supported in part by USDOE grant DE F603 91ER40674

$f$ Supported by the Deutsche Forschungsgemeinschaft

$g$ Supported by the Swedish Natural Science Research Council

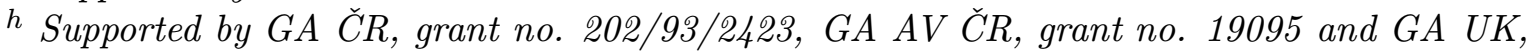
grant no. 342

i Supported by the Swiss National Science Foundation 


\section{Introduction}

The interaction of almost real photons and protons is the dominant process at the electronproton collider HERA. A small fraction of these events have large transverse energy $E_{T}$ in the hadronic final state, measured with respect to the electron-proton beam axis, or show the formation of jets.

The main characteristics of these events can be described by perturbative QCD calculations which are usually based on one hard parton-parton scattering per event. Two kinds of processes contribute to the high- $E_{T}$ jet production in photon-proton collisions (Fig. 1): (i) direct photon processes, where the photon couples directly to a parton in the proton, and (ii) resolved photon processes, in which the scattering occurs between a parton from the photon and one in the proton. Predictions for cross sections are obtained by the convolution of the matrix elements for the parton scattering with the parton distributions in the photon and the proton. Comparisons of measured cross sections with QCD calculations can provide important information on the parton scattering processes [1].

Comparisons of HERA data and QCD calculations revealed that the observed jets are not well described by such calculations, even if they include phenomenological models for the QCD radiation effects and the fragmentation phase. The energy flow adjacent to the jets - the so called underlying event energy, or jet pedestal - was found to be far above the QCD expectations $[2,3,4,5]$. This underlying event energy is measured outside of jets and includes energy resulting from radiation effects of the hard scattered partons and energy from the fragmentation process of these partons and the two beam remnants, i.e. the spectator partons. If interactions between the spectator partons occur in addition to the hard parton scattering, they also add to the energy level of the underlying event. If this underlying event energy is integrated into the measured jet energy, it alters drastically the measurement of the jet production rate. These effects have to be understood before drawing conclusions on the parton processes based on the comparison of measured jet cross sections with QCD calculations.

An excess of the measured underlying event energy above the QCD calculations has previously been observed in high energy $\bar{p} p$ collisions [6]. The data could be described by adding interactions of the beam remnants to the calculations [7]. Since the dynamics of hadronic final state production in photon-proton interactions is expected to be similar to that in hadron-hadron collisions, it is interesting to test such models in high energy photoproduction at HERA [8, 4].

( a )

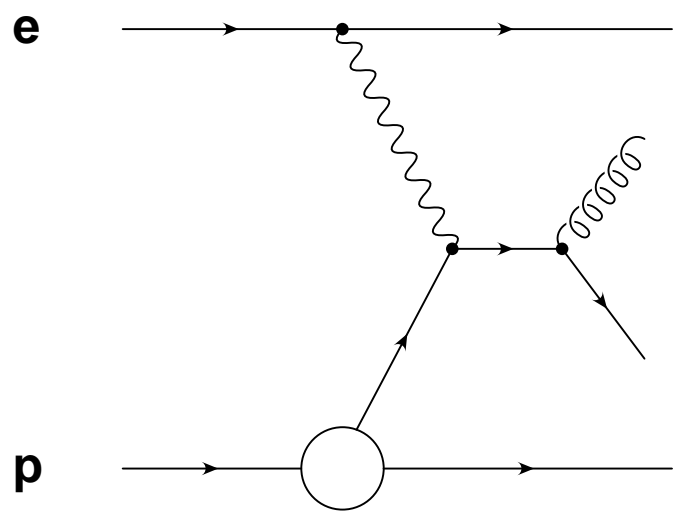

(b)

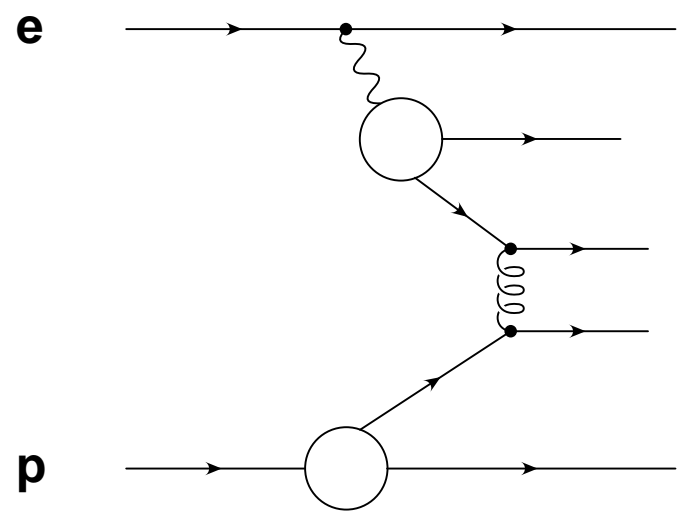

Figure 1: Examples of diagrams for direct (a) and resolved photon (b) processes in electron-proton scattering. The resolved photon interactions can have - in addition to the parton scattering process shown - interactions of the spectator partons of the photon and the proton. 
The photon offers a unique probe for the study of the underlying event energy. Resolved photoproduction events can in principle involve interactions between spectator partons of the proton and the photon. They can be compared to direct photoproduction events, which by definition cannot have these additional interactions. Such comparisons give information on the underlying event, as will be shown below.

In this paper detailed studies of photoproduction events with large transverse energy are presented. Three topics are considered:

1. The $E_{T}$ distribution: differential cross sections $d \sigma / d E_{T}$ and energy flow in the $\gamma p$ centerof-mass system (cms) are analysed, as well as the energy fraction which is contained in jets.

2. Properties of the underlying event: the energy density outside the jets is determined depending on the fraction of the photon energy which is available to the hard scattering process. Correlations of the transverse energy are measured.

3. Jet shape and rate: properties of jets are analysed depending on the jet energy. Jet cross sections are presented and the influence of the underlying event on the cross sections is studied.

\section{Detector description and event selection}

A detailed description of the $\mathrm{H} 1$ apparatus can be found elsewhere [9]. The following briefly describes the components of the detector relevant to this analysis, which makes use of the calorimeters, the luminosity system and the central tracking detector.

The liquid Argon (LAr) calorimeter [10] extends over the polar angular range $4^{\circ}<\theta<153^{\circ}$ with full azimuthal coverage, where $\theta$ is defined with respect to the proton beam direction $(+z$ axis). The calorimeter consists of an electromagnetic section with lead absorbers, corresponding to a depth of between 20 and 30 radiation lengths, and a hadronic section with steel absorbers. The total depth of the LAr calorimeter varies between 4.5 and 8 hadronic interaction lengths. The calorimeter is highly segmented in both sections with a total of around 45000 cells. Test beam measurements of LAr calorimeter modules have demonstrated energy resolutions of $\sigma(E) / E \approx 0.12 / \sqrt{E} \oplus 0.01$ with $E$ in $\mathrm{GeV}$ for electrons [11] and $\sigma(E) / E \approx 0.5 / \sqrt{E} \oplus 0.02$ for charged pions [12]. The hadronic energy scale and resolution have been verified from the balance of transverse momentum between hadronic jets and the scattered electron in deep inelastic scattering events and are known to a precision of $5 \%$ and $10 \%$ respectively.

The backward electromagnetic calorimeter (BEMC), with a thickness of 22.5 radiation lengths, covers the region between $151^{\circ}<\theta<177^{\circ}$. A resolution of $0.10 / \sqrt{E} \oplus 0.03$ with $E$ in $\mathrm{GeV}$ has been achieved for electrons. The BEMC energy scale is known to an accuracy of $1.7 \%$.

The calorimeter is surrounded by a superconducting solenoid providing a uniform magnetic field of $1.15 \mathrm{~T}$ parallel to the beam axis in the tracking region. Charged particle tracks are measured in two concentric jet drift chamber modules (CJC), covering the polar angular range $15^{\circ}<\theta<165^{\circ}$, and a forward tracking detector (FTD), covering the range $7^{\circ}<\theta<25^{\circ}$. The CJC is interleaved with inner and outer layers of multi-wire proportional chambers, which were used in the trigger to select events with charged tracks pointing to the interaction region.

The luminosity system consists of two $\mathrm{TlCl} / \mathrm{TlBr}$ crystal calorimeters having a resolution of $\sigma(E) / E=0.1 / \sqrt{E}$ with $E$ in GeV. The electron tagger is located at $z=-33 \mathrm{~m}$ and the 
photon detector at $z=-103 \mathrm{~m}$. The electron tagger accepts electrons with an energy fraction between 0.2 and 0.8 with respect to the beam energy and scattering angles below $\theta^{\prime} \leq 5 \mathrm{mrad}$ $\left(\theta^{\prime}=\pi-\theta\right)$.

The events used in this analysis were taken during the 1993 running period, in which 26.7 $\mathrm{GeV}$ electrons collided with $820 \mathrm{GeV}$ protons in HERA. The scattered electron was measured in the electron detector of the luminosity system. This requirement restricts the negative squared four momentum transfer of the photon to below $Q^{2}<0.01 \mathrm{GeV}^{2}$. Events were selected if they fulfilled the following criteria:

1. The energy deposited in the electron tagger was in the range $8 \leq E_{t a g} \leq 20 \mathrm{GeV}$. This interval corresponds to scaled photon energies $y=E_{\gamma} / E_{e}$ between $0.25 \leq y \leq 0.7$.

2. At least one charged particle was measured in the central tracker with transverse momentum above $0.3 \mathrm{GeV}$ coming from the interaction region.

3 . The reconstructed event vertex lay within $\pm 3 \sigma$ of the nominal interaction position along the beam axis. The width of the vertex distribution along the beam axis was approximately Gaussian with $\sigma=10 \mathrm{~cm}$.

Three different event samples were selected:

a) A sample of events which fulfills the requirements 1,2,3 is called 'minimum bias sample'. This sample is only used for comparisons with results obtained with the following two samples (see sections 4.2 and 5.1).

b) An event sample with large transverse energy in the final state (the 'high- $E_{T}$ sample') was defined by $E_{T} \geq 20 \mathrm{GeV}$ in the pseudo-rapidity range $-0.8 \leq \eta \leq 3.3$. It contained 3254 events and is used in sections 4.1, 4.2, 4.3 and 5.2.

c) Events with at least one jet as defined below (the 'jet sample'). This sample contained 3499 events and is used in sections 5.1, 6.1 and 6.2.

The pseudo-rapidity in the laboratory system is calculated via $\eta=-\ln (\tan (\theta / 2))$. The pseudorapidity in the photon-proton cms is calculated via $\eta^{*}=\eta-0.5 \ln E_{p} / E_{\gamma}$, where $\eta-\eta^{*} \approx 2$. The jet reconstruction was based on purely calorimetric measurements using a cone algorithm [13] in a grid of cells in the azimuthal angle $\varphi$ and laboratory pseudo-rapidity $\eta$ which extends from $-2 \leq \eta \leq 3$. The cone radius $R=\sqrt{\Delta \eta^{2}+\Delta \varphi^{2}}$ was chosen to be $R=1.0$. Each jet has transverse energy above $E_{T}^{\text {jet }} \geq 7 \mathrm{GeV}$ and lies in the pseudo-rapidity range $-1 \leq \eta^{\text {jet }} \leq 2.5$.

The events of samples a) and b) were triggered by a coincidence of an electron tagger signal and a charged particle measured in the central proportional chambers. For all jet analyses, sample c), also one track from the central jet chamber trigger was required. Background from non-ep scattering is negligible in all samples. The integrated luminosity for events with (without) jet requirement corresponds to $290(117) \mathrm{nb}^{-1}$ determined with an accuracy of $\pm 5 \%$.

\section{QCD generators}

For comparisons with the data three different event generators were used. They are based on tree-level QCD matrix elements. All calculations were done with the same parton distributions: for the proton structure the GRV-LO [14] leading order parton density parameterizations were used, and for the photon structure the GRV-LO [15] leading order parameterizations. As will be seen below, the generators can be grouped into those which include - in addition to the hard parton scattering - interactions of the beam remnants and those which do not. The models for the remnant interactions are based on parameterizations of results from hadron scattering, or (semi- ) hard scattering between spectator partons, or soft and (semi- ) hard parton scattering processes between spectator partons. 


\section{$3.1 \quad$ PYTHIA}

The PYTHIA 5.7 event generator for photon-proton interactions [16] was used together with a generator for quasi-real photons. PYTHIA is based on leading order (LO) QCD matrix elements and includes initial and final state parton radiation calculated in the leading logarithm approximation. The strong coupling constant $\alpha_{s}$ was calculated in first order QCD using $\Lambda_{Q C D}=200$ $\mathrm{MeV}$ with 4 flavours. The renormalization and factorization scales were both set to the transverse momentum $p_{t}$ of the partons emerging from the hard interaction. Since the QCD calculation of a hard parton scattering process is divergent for processes with small transverse momenta $p_{t}$, the requirement $p_{t} \geq p_{t}^{\text {min }}=2 \mathrm{GeV}$ was made. For the fragmentation process the LUND fragmentation scheme was used (JETSET 7.4 [17]). In this PYTHIA calculation the underlying event energy is generated by initial and final state parton radiation, and by fragmentation effects.

Within PYTHIA, interactions additional to the primary parton-parton scattering may be generated, so-called 'multiple interactions' $[7,8]$. These are calculated as LO QCD processes between partons from the photon and proton remnants. Multiple parton scattering has been studied in proton-antiproton collisions before $[19,18,20]$. The PYTHIA version extends the concept of the hard perturbative QCD parton scattering to the low transverse momentum, or semi-hard interaction region. As mentioned above, in this low $p_{t}$ region the LO parton scattering cross section $\sigma_{\text {parton }}$ diverges and becomes larger than the measured non-diffractive photoproduction cross section $\sigma_{n d}$. Since in the multiple interaction model each of the two incoming beam particles may be viewed as a beam of partons, the problem of too large parton cross sections can be solved by allowing for several parton scattering processes within one observable $\gamma p$ event. To prevent a rapid rise of the jet cross section at small transverse jet energies, a unitarization scheme has been introduced which results in the damping of the cross section at small parton momenta. In this way, the calculated hadronic cross section can stay below the non-diffractive $\gamma p$ cross section.

In the simplest version of the PYTHIA multiple interaction model used here, the transverse momentum cut-off of the hard interactions is lowered to $p_{t}^{\text {mia }}<p_{t}^{\text {min }}$. The mean number of (semi-) hard interactions is given by $\langle n\rangle=\sigma_{\text {parton }}\left(p_{t}^{m i a}\right) / \sigma_{n d}$, the fluctuations are calculated from a Poisson distribution. The number of additional interactions is typically of order $1-2$. The parton process with the highest transverse momentum in the partonic final state can be given by any quark or gluon matrix element. This process includes then initial and final state parton radiation effects and its partons are connected to the beam remnants by strings. The additional parton scattering processes in the event are calculated as perturbative gluon-gluon scattering processes. The initial state gluon momenta of each subsequent process are related to the remaining energy of the beam remnants. The resulting fractional momenta are used to determine the parton densities of the beam remnants.

The additional interactions contribute significantly to the transverse energy flow in the event. This is mainly influenced by the average number of interactions per event, the cut-off $p_{t}^{m i a}$, and by the structure functions used for the calculations. Using the GRV parameterizations for the parton distributions of the photon and the proton, an adjustment of the transverse momentum cut-off to $p_{t}^{\text {mia }}=1.2 \mathrm{GeV}$ resulted in an adequate description of the energy flow next to jets as observed in the data (see section 5.1). The PYTHIA version with multiple interactions was used to determine corrections for apparatus inefficiencies.

\subsection{PHOJET}

The PHOJET 1.0 event generator was designed to simulate in a consistent way all components which contribute to the total photoproduction cross section [21]. It is based on the twocomponent Dual Parton Model [22]. The implementation of the PHOJET generator is similar 
to the Monte Carlo event generator DTUJET [23] which simulates multi-particle production in high energy hadron collisions. This latter generator was originally intended for the description of soft hadronic interactions and was then extended to hard scattering processes. In contrast to PYTHIA, PHOJET incorporates both, multiple soft and hard parton interactions, on the basis of a unitarization scheme [24]. The soft hadronic processes are described by the soft, 'supercritical' Pomeron [25]. These processes are simulated by a two-string ansatz which allows for initial transverse momenta of the partons at the ends of the strings. The hard processes are calculated using the LO QCD matrix elements. Final state parton radiation effects are simulated using the JETSET 7.4 program [17]. Hard initial state parton radiation is not included in this version of PHOJET. The lower momentum cut-off for hard parton interactions was set to $p_{t}^{\text {min }}=3 \mathrm{GeV}$. Due to the unitarization scheme, small variations of this cut-off parameter do not have a large influence on the results of this generator. The model parameters which describe the soft part of the $\gamma p$ interactions have been tuned using results from proton-antiproton collisions and low energy photoproduction cross section measurements. For the fragmentation the LUND string concept is applied (JETSET $7.4[17]$ ).

\subsection{HERWIG}

The HERWIG $5.8 \mathrm{ep}$ generator is also based on the LO QCD calculations [26]. This program was designed to have as much input from perturbative QCD as possible, in order to minimize the free parameters. HERWIG includes a parton shower model which allows for interference effects between the initial and final state showers (colour coherence) [27]. The renormalization and factorization scales were set according to the transverse momentum of the scattered partons with a lower cut-off used around $p_{t}^{\min } \geq 2 \mathrm{GeV}$. The strong coupling constant $\alpha_{s}$ was calculated to first order using $\Lambda_{Q C D}=180 \mathrm{MeV}$ for 5 flavours. A cluster model is used to simulate the hadronization effects [28].

HERWIG allows optionally for additional interactions of the beam remnants. These interactions are called soft underlying event and are parameterizations of experimental results on 'soft' hadron-hadron collisions. A tuning of the strength and frequency parameters is still in progress. Recently, also a model for multiple parton interactions has been developed for HERWIG [29] which has not been used in this paper.

\section{Distributions of transverse energy}

\subsection{Transverse energy cross section}

Cross sections as a function of the total transverse event energy $E_{T}$ have previously been measured in $\gamma p$ [30] and $\bar{p} p$ scattering at different $\mathrm{cm}$ energies [31, 33, 34]. They fall steeply in the low $E_{T}$ region (soft hadronic interactions) and tend to flatten towards large transverse energy. For sufficiently high $E_{T}$ the distributions can be equally well described by either a power law $\left(E_{T}\right)^{-n}$ or an exponential decrease $\exp \left(-\lambda E_{T}\right)$.

In Fig. 2a the measured differential ep cross section $d \sigma / d E_{T}$ is shown. For this analysis the high- $E_{T}$ event sample, defined in section 2 , was used. The transverse energy was integrated over the cms pseudo-rapidity range $-2.5 \leq \eta^{*} \leq 1$. This region is fully covered by the electromagnetic and hadronic sections of the LAr calorimeter. The measurement corresponds to scaled photon energies between $0.3 \leq y \leq 0.7$ and negative squared momentum transfer $Q^{2} \leq 0.01 \mathrm{GeV}^{2}$.

Corrections for apparatus inefficiencies applied to the observed transverse energy were parameterized as a function of the pseudo-rapidity of the energy deposits. They decrease with 

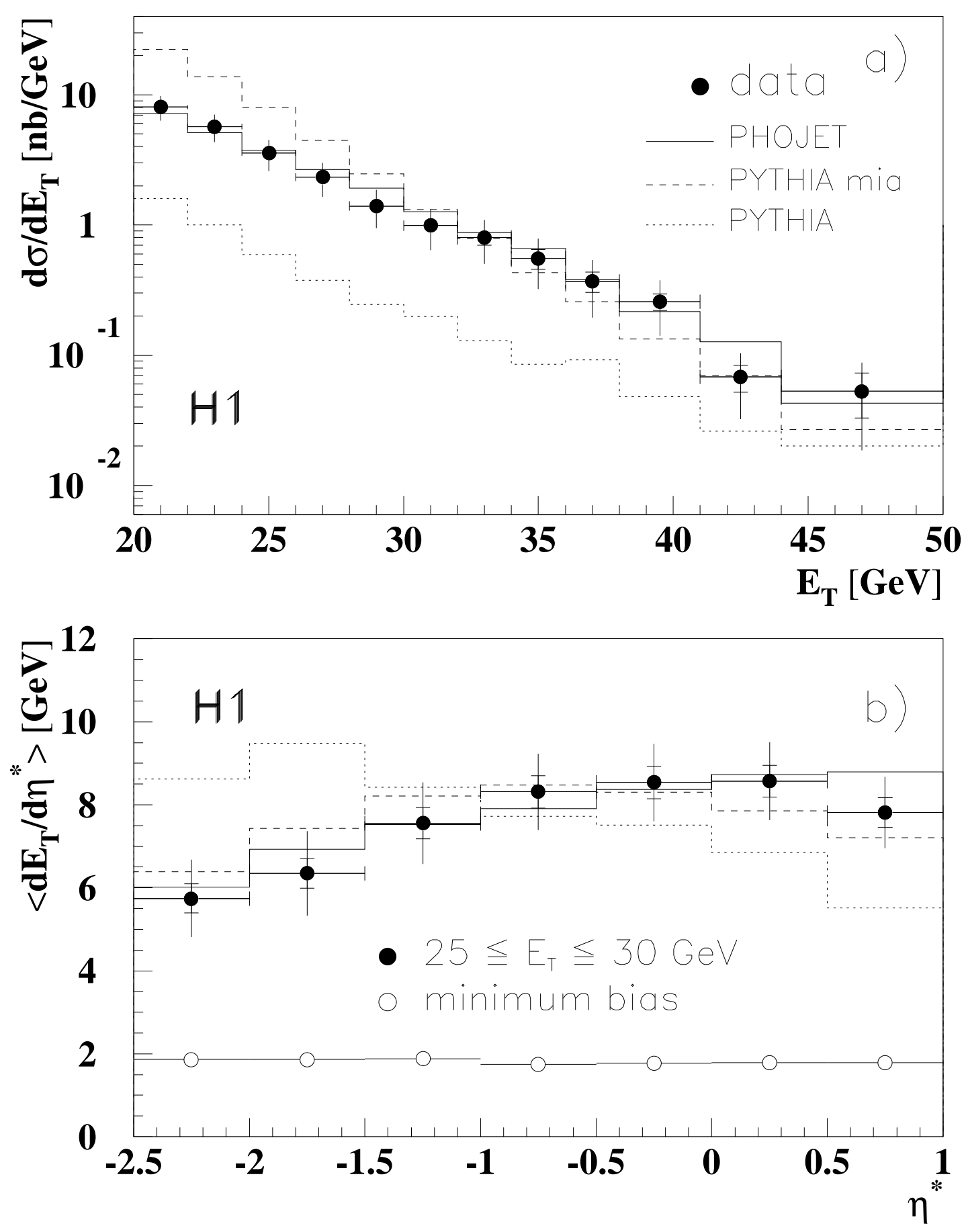

Figure 2: a) The differential transverse energy ep cross section integrated in the pseudo-rapidity interval $-2.5 \leq \eta^{*} \leq 1$. Full circles are data, the inner error bars are statistical, the outer error bars are the quadratic sum of statistical and systematic errors. The histograms show the calculations of $Q C D$ generators with interactions of the beam remnants (full=PHOJET, dashed=PYTHIA) and without them (dotted $=$ PYTHIA).

b) Corrected transverse energy flow per event versus the pseudo-rapidity in the $\gamma p$ cms, where $\eta^{*}>0$ refers to the proton direction. Full circles refer to events with total transverse energies between $25 G e V \leq$ $E_{T}\left(-2.5 \leq \eta^{*} \leq 1\right) \leq 30 \mathrm{GeV}$. Open circles refer to minimum bias data. Histograms are as in a). 
pseudo-rapidity and amount to factors between $1.2-0.8$. The corrections depend on the Monte Carlo model used to determine them. This leads to a contribution of $10-15 \%$ to the systematic error, increasing with decreasing pseudo-rapidity. Corrections were applied for the acceptance of the electron tagger and these contribute $5 \%$ to the systematic error. This error also includes the uncertainty of the luminosity measurement. The determination of the trigger efficiency has a $2 \%$ error. The dominant systematic error comes from the uncertainty in the knowledge of the LAr calorimeter energy scale. This error increases with $E_{T}$ and amounts to $15-50 \%$.

The differential cross section in Fig. 2a is compared to different QCD calculations: the PYTHIA generator without multiple interactions (dotted histogram) gives too small values of the cross section. The HERWIG generator gives similar low values (not shown). The PYTHIA calculation with multiple interactions (dashed histogram) gives too large cross section values at $E_{T}=20 \mathrm{GeV}$, but is compatible with the data in the large $E_{T}$ region. The PHOJET generator, which also includes multiple interactions, provides a good description of the data (full histogram).

The shape of the measured differential $E_{T}$ cross section can be described by a power law with $n=5.9$ with a $2 \%$ statistical uncertainty in $n$. The power $n$ is similar to that of the transverse energy jet cross section [2]. Therefore the spectrum can be consistently interpreted by hard parton scattering processes. The data are in this $E_{T}$ range equally well described by an exponential function. An exponential fit gives the slope $\lambda=0.21$ at $\sqrt{s_{\gamma p}}=200 \mathrm{GeV}$ with a $5 \%$ statistical error in the fit.

\subsection{Transverse energy flow}

The peripheral scattering of two hadrons results in a transverse energy flow which can be described by a rapidity plateau of width $2 \ln (\sqrt{s} / m)$, with final state hadrons of mass $m$ carrying transverse momenta of around $p_{t} \approx 300 \mathrm{MeV}, \sqrt{s}$ being the cms energy [32]. In Fig. $2 \mathrm{~b}$ the average transverse energy flow in photoproduction events is shown versus the $\gamma p$ cms pseudo-rapidity $\eta^{*}$. The distributions are corrected for detector effects. The open circles refer to minimum bias data, defined in section 2. These data exhibit a plateau within the pseudo-rapidity interval shown, as expected from peripheral scattering of a hadron-like photon and a proton.

The full circles refer to the high- $E_{T}$ event sample, defined in section 2 , with corrected total transverse energies, summed in the pseudo-rapidity range $-2.5 \leq \eta^{*} \leq 1$, between $25 \mathrm{GeV} \leq$ $E_{T} \leq 30 \mathrm{GeV}$. The distribution is not compatible with being flat and increases towards the origin of the $\gamma p \mathrm{cms}$.

Similar distributions have been observed in $\bar{p} p$ scattering with large transverse energy in the final state [33]. Here the $E_{T}$ distribution versus pseudo-rapidity is centered on the cms rapidity

$\eta^{*}=0$ and has (e.g. at $\sqrt{s_{\bar{p}}}=630 \mathrm{GeV}$ ) a half width at half maximum of only one unit of rapidity for very large $E_{T} \geq 200 \mathrm{GeV}$ which is to be compared with a possible total plateau width of $\Delta \eta \sim 13$ (using the proton mass).

The observed increase in the $\gamma p \mathrm{cms}$ is, to some extent, unexpected: since there are relatively more partons at large momentum fraction $x$ in the photon than in the proton, one would naively expect this distribution to be peaked somewhere in the photon hemisphere, i.e. at negative rapidities. The PYTHIA calculation without multiple interactions (dotted histogram) confirms this expectation. A multiple interaction mechanism, however, adds additional energy around the origin of the $\gamma p \mathrm{cms}$. The superposition of parton scattering processes by a PYTHIA calculation with multiple interactions (dashed histogram) results in a description which is compatible with the data. Here the maximum is closer to $\eta^{*}=0$, but still shows a shift towards the photon hemisphere. The PHOJET calculation (full histogram), which also includes interactions of the beam remnants, provides a good description of the energy flow. 


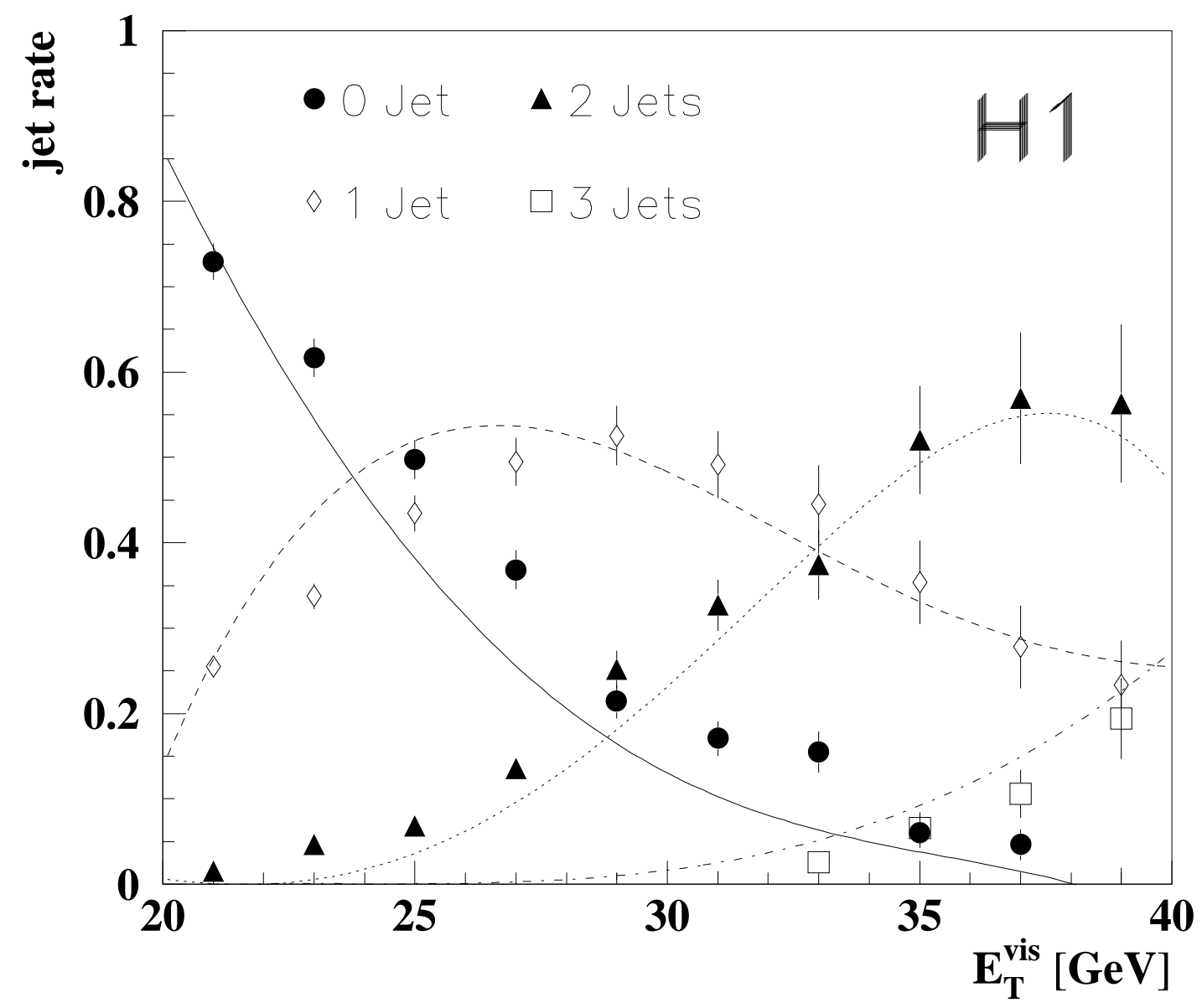

Figure 3: Observed relative rate of $0=$ circles, $1=$ diamonds, $2=$ triangles, $3=$ squares jet events versus the observed transverse energy collected in the pseudo-rapidity range $-3 \leq \eta^{*} \leq 1$. The observed jet energies $E_{T}^{\text {jet }}$ are above $7 \mathrm{GeV}$ summed in a cone of size $R=1$ for jet axes between $-2.5 \leq \eta^{*} \leq 0.5$. Symbols are data with only statistical error bars shown. The curves are calculations of a QCD generator including interactions of the beam remnants and a detailed simulation of the detector effects (PYTHIA).

\subsection{Jet rate}

In tree level QCD calculations two partons emerge from a hard scattering process, each fragmenting into a jet. The measured jet multiplicity depends, however, on the total transverse energy in the event and on the jet definition. Here a cone algorithm is used, with cone size $R=1$ and a transverse energy threshold $E_{T, \min }^{j e t}$.

In Fig. 3 the relative rates of the observed jet multiplicities $n_{j e t}$ are shown as a function of the uncorrected transverse event energy $E_{T}^{v i s}$. These results were obtained using the high- $E_{T}$ event sample. The visible jet energy threshold is set to $E_{T, \min }^{\text {jet }}=7 \mathrm{GeV}$ and the jet axis is required to be in the range $-2.5 \leq \eta^{*} \leq 0.5$. The transverse event energy is the summed transverse energy deposited in the pseudo-rapidity interval $-3 \leq \eta^{*} \leq 1$.

At $E_{T}^{v i s}=20 \mathrm{GeV}, 70 \%$ of the events have no jets at all, given the above definition, while at $E_{T}^{v i s}=35 \mathrm{GeV}$, half of the events contain two jets. The two jet configurations are expected from the tree level QCD picture. At $E_{T}^{v i s}=40 \mathrm{GeV}$ more than $10 \%$ three jet events are observed, 
showing the effect of higher order processes with possible contributions from the photon remnant. At this $E_{T}^{v i s}$ there are essentially no events without jets.

The average fraction of the total visible event transverse energy which is contained in jets with the chosen parameters was calculated at $E_{T}^{v i s}=20 \mathrm{GeV}$ to be $\sim 10 \%$. At $E_{T}^{v i s}=40 \mathrm{GeV}$ about half of the transverse event energy is contained in jets.

The jet rates are by and large described by calculations of the PYTHIA generator with multiple interactions, which include here a detailed simulation of detector effects (Fig. 3). PYTHIA without multiple interactions gives much too large jet rates: at $E_{T}^{v i s}=30 \mathrm{GeV}$ the contribution of events without jets has already vanished, while the relative two-jet rate is two times the rate of the data (not shown). The latter model comparison indicates that the energy depositions beyond those resulting from the tree level hard parton scattering process plus parton showers are neither correlated with the jets nor jet like, but 'soft' energy depositions spread throughout the event.

\section{Summary: Distributions of transverse energy}

The photoproduction data with large transverse energy in the final state show signatures of hard scattering processes: the slowly decreasing transverse energy spectrum, the pseudo-rapidity distribution which is not flat, and the multi-jet production all demonstrate the scattering of constituents of the photon and proton. Comparisons of the data with different generators which are based on LO QCD matrix elements plus parton showers - show that models which include interactions between the beam remnants provide considerably better descriptions of the data than models without such a mechanism.

\section{$5 \quad$ Energy of the underlying event}

\subsection{Transverse energy density outside of jets}

In QCD inspired models various processes contribute to the transverse energy flow outside jets (e.g. [6]):

A.) Transverse energy of the partons that participate in the hard scattering including 1) initial and 2) final state radiation from these partons,

B.) Transverse energy from the interactions between the spectator partons which is essentially uncorrelated with the hard scattering process,

C.) Transverse energy of non-interacting spectator partons which is also essentially uncorrelated with the hard parton scattering process.

In all three cases fragmentation effects have to be considered in addition. Monte Carlo generators which include all these components describe distributions of the hadronic final state in $\bar{p} p$ [7] and $\gamma p$ [4] scattering considerably better than generators including only the effects of the hard parton interaction (item A) and remnant fragmentation (item C). The clearest experimental evidence for item B would be hard spectator interactions. Their observation has been reported in analyses of multi-jet events in $p p$ and $\bar{p} p$ collisions $[18,20]$, but were not confirmed by another $\bar{p} p$ experiment [19]. 
At HERA the photon offers a unique probe to study the underlying event: in $\gamma p$ interactions with resolved photons the energy flow outside jets should contain all three components mentioned above. Direct $\gamma p$ interactions, however, do not involve an initial state parton from the photon side, hence there is no initial state QCD radiation (item A.1). There are no spectator partons interactions (item B) and there is no photon remnant (item C). The direct processes should, however, have the same final state radiation (item A.2) as the resolved photon interactions. The different contributions can therefore partly be disentangled by studying the underlying event as a function of, e.g., the fractional momentum of the remnant from the photon side $\left(1-x_{\gamma}\right)$ which is 0 for direct photon interactions and greater than 0 in the case of resolved photon interactions.

The jet event sample is used (defined in section 2), requiring at least two jets. To avoid a miss-interpretation of the photon remnant as a hard jet the pseudo-rapidity difference between the two jets had to be below $\Delta \eta \leq 1.2$. The momentum fraction of the parton from the photon can be estimated from

$$
x_{\gamma}^{j e t s}=\frac{E_{T}^{j e t 1} e^{-\eta^{j e t 1}}+E_{T}^{j e t 2} e^{-\eta^{j e t 2}}}{2 E_{\gamma}}
$$

where $x_{\gamma}^{j e t s}$ is reconstructed using the two jets with the highest transverse energy $E_{T}^{j e t}$ in the event and their rapidities $\eta^{j e t}$. The energy of the photon $E_{\gamma}$ is determined from the energy measured in the electron tagger. The resolution of $x_{\gamma}^{\text {jets }}$ is between $15-30 \%$, getting worse as $x_{\gamma}^{\text {jets }}$ increases from 0 to 1 .

In this section the measurement of the energy density outside jets as a function of $x_{\gamma}^{\text {jets }}$ is discussed. The transverse energy is summed in the central rapidity region of the $\gamma p$ collision $-1 \leq \eta^{*} \leq 1$. Energy deposited around the two jet axes within $R=\sqrt{\Delta \varphi^{2}+\Delta \eta^{2}} \leq 1.3$ is excluded from the energy summation. The transverse energy density $<E_{T}>/(\Delta \eta \Delta \varphi)$ is then defined as the energy sum $\left\langle E_{T}\right\rangle$ per unit area in the $(\eta, \varphi)$ space averaged over all events in the sample (Fig. 4). The distribution was corrected for detector effects. It rises towards small values of $x_{\gamma}^{\text {jets }}$.

Systematic errors originate from the following sources: the observed energy was corrected for detector effects, the correction used depends on the pseudo-rapidity of the energy deposit. There is a small dependence on the Monte Carlo model used to obtain the correction which contributes $10 \%$ to the systematic error. Migration effects in $x_{\gamma}^{\text {jets }}$ were determined with different generator models and lead to an uncertainty in the energy density at the level of $14 \%$. These errors are shown in Fig. 4. In addition, the uncertainty in the knowledge of the hadronic energy scale of the LAr calorimeter may change the overall normalization of the energy density by $5 \%$. Contributions from other sources of systematic errors are negligible.

The long-dashed line in Fig. 4 indicates the energy density found in minimum bias events where $x_{\gamma}^{j e t s}$ is not measurable. The energy density for directly interacting photons $\left(x_{\gamma}^{j e t s}=1\right)$ is found to be close to the energy density found in minimum bias events. This observation is consistent with a recent comparison of the measured transverse energy density in the central rapidity region of the $\gamma p$ collision. Here the same transverse energy density was measured in minimum bias data and deep inelastic scattering events (direct photons) [36]. These comparisons show that neither the initial state radiation effects of the parton from the proton, nor the final state parton radiation effects can be large. The measured energy density increases towards small $x_{\gamma}^{\text {jets }}$ by $0.6 \mathrm{GeV} / \mathrm{rad}$ to 3.5 times the minimum bias energy density. It is unlikely that such a large effect is caused alone by initial state radiation of the parton from the photon side. In the QCD inspired picture described at the beginning of this section, the natural explanation for the enhanced energy flow is interactions between the parton spectators (item B): the probability for these additional interactions increases with the energy of the photon remnant.

Also shown in Fig. 4 are the results of several different Monte Carlo generators. The dotted histogram refers to the PYTHIA calculation without multiple interactions. This calculation is 


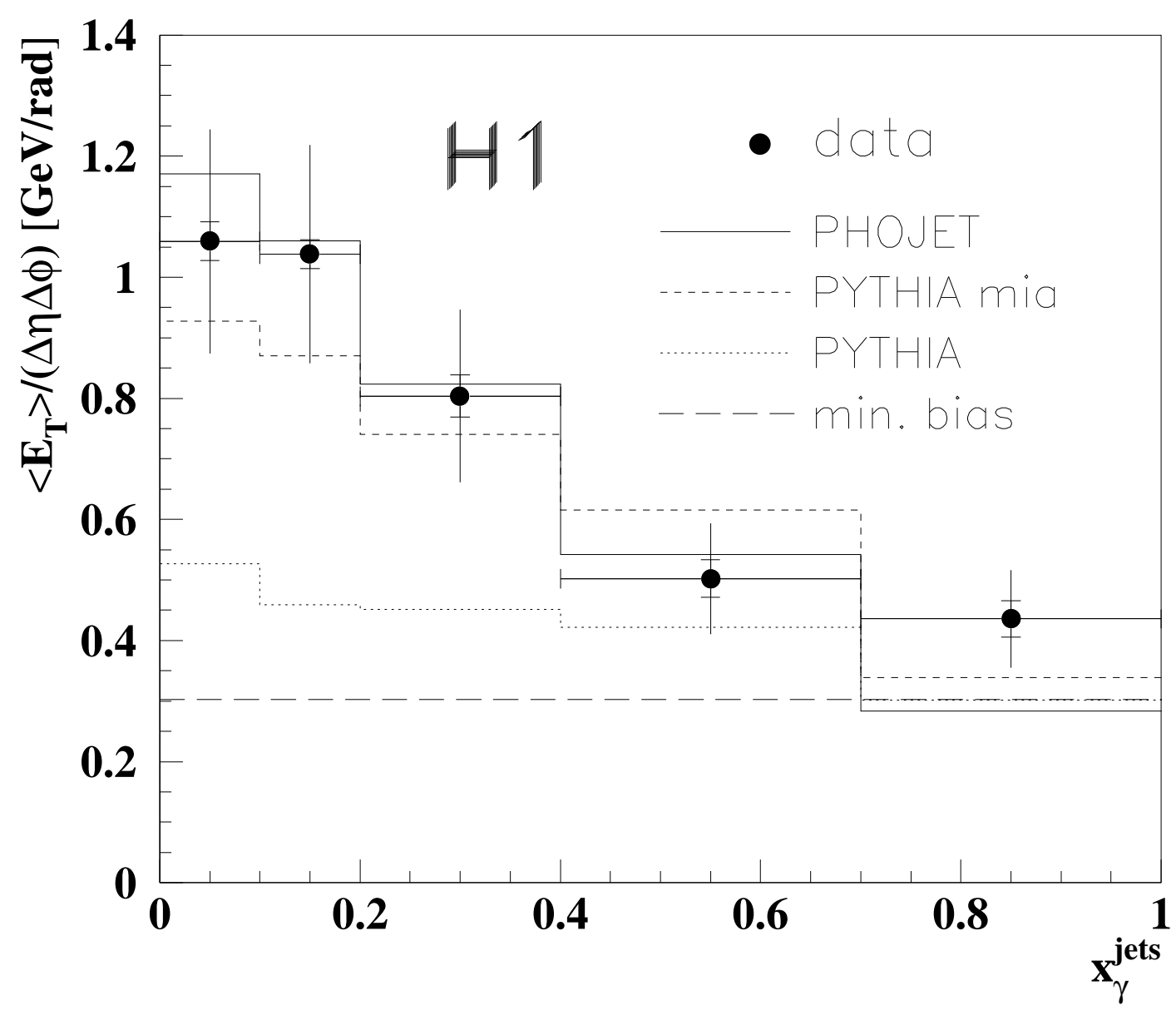

Figure 4: Corrected transverse energy density in the central rapidity region of the $\gamma p$ collision $\left|\eta^{*}\right|<1$ per event and unit area in pseudo-rapidity and $\varphi$ space, outside of the two jets with the highest $E_{T}^{\text {jet }}$. The variable $x_{\gamma}^{j e t s}$ is a measure of the momentum fraction of the parton from the photon side. Circles are data, the inner error bars are statistical, the outer error bars result from the quadratic sum of statistical and systematic errors. An overall uncertainty in the hadronic energy scale of $5 \%$ is not shown in the figure. The long-dashed line indicates the energy density measured in minimum bias events. The histograms show the calculations of different $Q C D$ generators with interactions of the beam remnants (full=PHOJET, short-dashed=PYTHIA), and without them (dotted=PYTHIA). 
compatible with the measured energy density at large $x_{\gamma}^{\text {jets }}$, but shows only a small increase towards $x_{\gamma}^{j e t s}=0$, as one may expect from increased initial state radiation effects. The use of another parameterization of the photon structure function, e.g. LAC1 [37], does not change this result (not shown). The HERWIG generator gives similar results (not shown). The QCD simulations without multiple interactions show that the observed increase cannot be understood as a kinematical bias.

Adequate descriptions of the data are provided by calculations which include interactions between the photon and proton spectator partons, PYTHIA with multiple interactions (shortdashed histogram) and PHOJET (full histogram). Using PYTHIA with multiple interactions together with the LAC1 structure function parameterization instead of the GRV-LO set increases the number of multiple interactions per event due to the larger gluon density at small fractional momenta $x_{\gamma}$. A re-adjustment of the momentum cut-off from $p_{t}^{\text {mia }} \geq 1.2 \mathrm{GeV}$ to $p_{t}^{\text {mia }} \geq 2 \mathrm{GeV}$ results again in an adequate description of the measured energy density.

For the first time the underlying event energy has been measured in jet events using direct and resolved photon probes. The large difference of the energy density in the central rapidity region of the $\gamma p$ collision between direct and hadron like photons and the comparisons with different Monte Carlo models demonstrate that an additional source of $E_{T}$ is probably needed beyond that coming from a two parton scattering process and the according parton radiation effects.

\subsection{Transverse energy correlations}

Energy-energy correlations are sensitive measures of how energy is distributed over the available phase space. In this section we examine further the transverse event energy measured in Fig. 2 and in jet events (Fig. 4). A study of the rapidity correlations relative to the central rapidity region of the $\gamma p$ collision, where the event energy is largest, should provide information on the underlying event and is an important test of the models which describe the average event energy correctly.

In Fig. 5 rapidity correlations $\Omega$ are shown with respect to the $\gamma p$ cms pseudo-rapidity $\eta^{*}=0$ for the high- $E_{T}$ event sample, defined in section 2. Energy deposits within jets are included in this measurement. The correlation function $\Omega$ was defined as

$$
\Omega\left(\eta^{*}\right)=\frac{1}{N_{e v}} \sum_{i=1}^{N_{e v}} \frac{\left(<E_{T, \eta^{*}=0}>-E_{T, \eta^{*}=0, i}\right)\left(<E_{T, \eta^{*}}>-E_{T, \eta^{*}, i}\right)}{\left(E_{T}^{2}\right)_{i}} .
$$

Here $E_{T}$ is the total transverse energy measured in the range $-3.1<\eta^{*}<1.3$, and the other terms refer to transverse energies measured in the bins of size $\Delta \eta=0.22$. The average value of the transverse energy in a bin $\left\langle E_{T, \eta^{*}}\right\rangle$ was determined from all events in the sample.

The data distribution shows short range correlations around the $\gamma p$ cms reference bin at $\eta^{*}=0$. Long range anti-correlations are observed in the photon hemisphere. These have a minimum around $\eta^{*}=-1.8$ and disappear around $\eta^{*} \approx-3$.

The dotted histogram in Fig. 5 shows the calculation of the PYTHIA generator without multiple interactions, including a detailed simulation of the detector effects. The correlations have the correct shape, but the (anti-) correlation is twice as strong as observed in the data. This implies that too much energy in the central rapidity region of the $\gamma p$ collision is correlated with the other energy deposited in the event. The PYTHIA version including multiple interactions is shown in Fig. 5 as dashed histogram. Here event energy is added around $\eta^{*}=0$ (Fig. 2b). This stems from the interactions of the spectator partons and is not correlated with the major part of the event energy, which results from the primary hard scattering process. Although the 


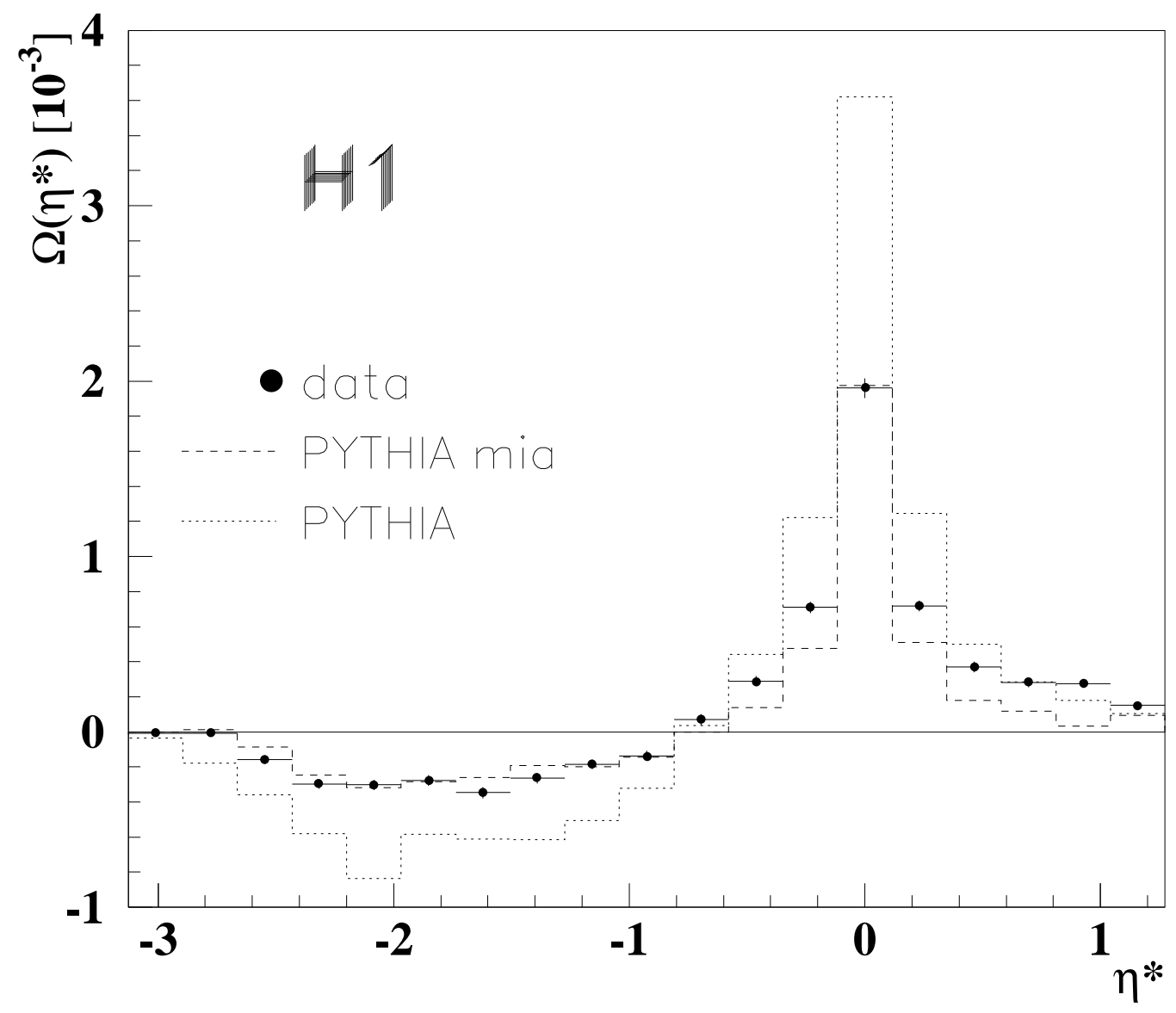

Figure 5: Observed rapidity correlations with respect to the central rapidity region of the $\gamma p$ collision $\eta^{*}=0$. Full circles are data, only statistical error bars are shown. The dashed (dotted) histogram represents a calculation of the QCD generator PYTHIA with (without) interactions of the beam remnants. 
model does not give a perfect description of the data, it shows that the addition of uncorrelated energy to the events results not only in the correct average underlying event energy (Fig. 4), but also gives the correct correlation strength (Fig. 5). The same conclusions hold for an event sample where a jet is explicitly required.

\section{Summary: Energy of the underlying event}

There are large differences in the underlying event transverse energy density in $\gamma p$ collisions dependent on whether the photons involved are direct or hadron like. In the central rapidity region of the $\gamma p$ collision, processes where large fractions $x_{\gamma} \approx 1$ of the photon energy are carried into the hard scattering process have a similar transverse energy density as minimum bias events. Processes with resolved photons $x_{\gamma} \approx 0$ are found to have 3.5 times the transverse energy of minimum bias events. This increase is much larger than expected from QCD generators using LO QCD matrix elements plus parton showers, but can be explained by models including interactions between the beam remnants. In addition, comparisons of the measured energyenergy correlations with different models demonstrate that the additional energy of the beam remnant interactions decreases the correlation strength to the level observed in the data.

Therefore, the underlying event in photoproduction events can be consistently interpreted as the superposition of a hard scattering process plus interactions between the beam spectators. The characteristic kinematic quantity is the momentum fraction of the parton from the photon side. This determines the energy in the hard interaction and the energy left for interactions of the two beam remnants.

\section{Properties of jets and jet cross sections}

\subsection{Jet shape}

The distribution of transverse energy around a jet axis shows an approximately Gaussian shape. In perturbative QCD the width of these jets is expected to decrease with increasing jet energy.

In Fig. 6a the uncorrected transverse energy flow in the azimuthal direction $\Delta \varphi$ around the jet with the highest $E_{T}^{j e t}$ in events with at least two jets is shown. The jets were found in the photon hemisphere between $-2 \leq \eta_{\text {jet }}^{*} \leq-1$ where detector corrections are small. The transverse jet energies were between $9 \leq E_{T}^{\text {jet }} \leq 11 \mathrm{GeV}$ summed in a cone of size $R=1$. The energy flow was integrated in a slice of $\left|\eta^{*}-\eta_{j e t}^{*}\right| \leq 1$ around the jet axis. The two jets usually deviate from a back-to-back configuration in the azimuthal angle. The azimuthal angle between the two jets was defined such that $-\pi \leq \Delta \varphi \leq 0$. Since the jets have a total width of approximately two units in the azimuthal direction (Fig. 6a), the region $1 \leq \Delta \varphi \leq 2$ is essentially free of the second jet and shows the underlying event energy.

The full curve in Fig. 6a represents an empirical 3 parameter fit of the jet profile using a function closely related to a Gaussian:

$$
f(\Delta \varphi)=A \exp \left(-(\sqrt{|\Delta \varphi|}+b)^{4}+b^{4}\right)+P
$$

Parameter $A$ describes the amplitude of the jet at $\Delta \varphi=0$ which is mainly constrained by the energy measured in the central bin. $P$ reflects the underlying event energy (jet pedestal). The full width at half maximum above the pedestal is then $\Gamma=2\left(\left(\ln 2+b^{4}\right)^{1 / 4}-b\right)^{2}$. Regions potentially affected by the second jet, $\Delta \varphi<-1$ and $\Delta \varphi>2.2$, are excluded from the fit. 

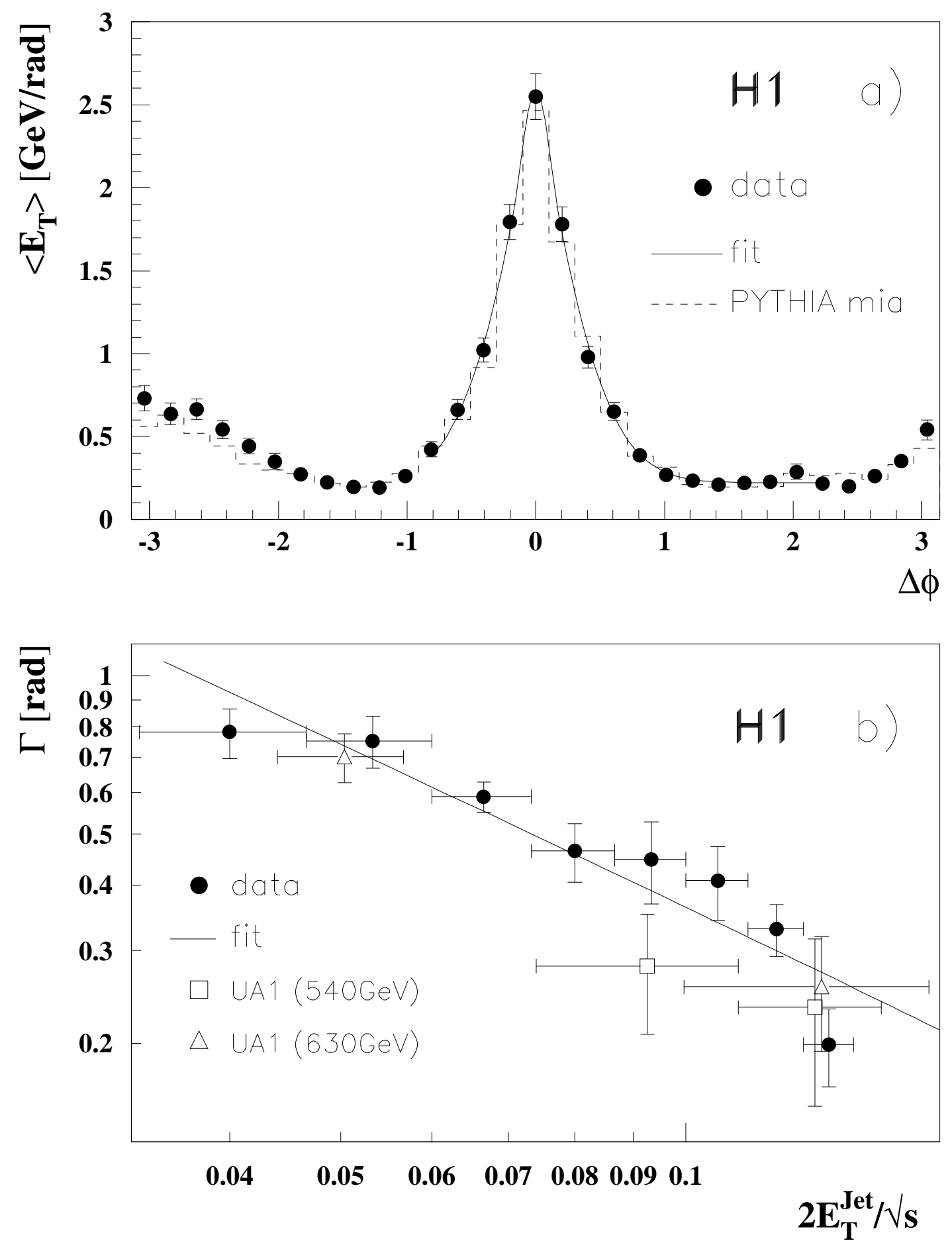

Figure 6: a) Observed transverse energy around the jet axis is shown versus the azimuthal angle $\Delta \varphi$ for jets in the photon hemisphere $-2 \leq \eta_{j e t}^{*} \leq-1$ and $9 \leq E_{T}^{j e t} \leq 11 \mathrm{GeV}$. The full circles represent the data with their statistical error bars. The axis of the second jet is between $-\pi \leq \Delta \varphi \leq 0$. The histogram is a calculation of the QCD event generator PYTHIA with multiple interactions, and the curve is a fit using eq. (3).

b) The fitted full width $\Gamma$ at half maximum above the underlying event of a jet is shown depending on the scaled jet energy $2 E_{T}^{\text {jet }} / \sqrt{s_{e p}}$, where the ep center-of-mass energy is used following [38]. Full circles are $H 1 \mathrm{\gamma p}$ data, the error bars represent the quadratic sum of the statistical and systematic errors. Open symbols are derived from fits to jet profiles of $\bar{p} p$ data using the cms energies $\sqrt{s_{\bar{p}}}$ [39, 40]. The full line represents a $1 / E_{t}^{\text {jet }}$ fit to the $\gamma p$ data. 
In Fig. $6 \mathrm{~b}$ the width $\Gamma$ of jets from $\gamma p$ interactions (full circles) is shown as a function of the scaled transverse jet energy $2 E_{T}^{j e t} / \sqrt{s_{e p}}$. Here the $e p \mathrm{cms}$ energy is used, following a QCD analysis of jet shapes [38]. The decrease of $\Gamma$ for jet energies between $5 \leq E_{T}^{\text {jet }} \leq$ $20 \mathrm{GeV}$ is clearly seen. This effect is well described by the QCD generators, although it is not inconceivable that other models could explain this effect. The decrease can be described by a $1 / E_{T}^{j e t}$ dependence. Such a dependence is expected from a QCD analysis presented in [38]. Within errors, no dependence of $P$ on $E_{T}^{\text {jet }}$ was found in this restricted pseudo-rapidity range. The amplitude $A$ varies as a function of $E_{T}^{\text {jet }}$ and $\Gamma$. Systematic errors in Fig. $6 \mathrm{~b}(\leq 10 \%)$ are dominated by the choice of the $\Delta \varphi$ interval used for the fit. Fits to the jet profiles in the rapidity projection give compatible results within error bars. Since the jet pedestal increases as a function of the rapidity in the $\eta$ region considered here, more fit parameters are needed to describe the data. This leads to larger error bars on the fitted jet width than in the case of the fits to the $\varphi$-projections of the jet profiles shown in Fig. $6 \mathrm{~b}$.

In the same Fig. $6 \mathrm{~b}$, the photoproduction data are compared to results from fits to jet profiles of $\bar{p} p$ data at different cms energies (open symbols) [39, 40]. Here pseudo-rapidity projections of the jet profiles were used, allowing $P$ in (3) to depend linearly on the rapidity. The same jet width as in $\gamma p$ data was measured in $\bar{p} p$ data at different jet energies. Within the error bars the jet widths found in $\gamma p$ and $\bar{p} p$ are compatible with having the same dependence on the ratio of the jet energy and the cms energy.

\subsection{Jet cross sections}

Differential inclusive jet cross sections have previously been measured in photoproduction events at HERA [2,3]. The jet cross section $d \sigma / d E_{T}^{j e t}$ as a function of transverse energy falls steeply and can be described by a power law $\left(E_{T}^{j e t}\right)^{-n}$. Such a falling distribution is expected from QCD calculations. Here the matrix elements of different parton scattering processes (quark-gluon, gluon-gluon etc.) are summed according to the quark and gluon distributions in the photon and the proton. The calculations are only modestly sensitive to the parton distributions, and reflect essentially features of the matrix elements. The measured cross section can be described by these calculations and can, therefore, be interpreted as the result of a parton scattering processes. The observed jets are mainly located in the photon hemisphere and cover there several units in pseudo-rapidity. Jet cross sections $d \sigma / d \eta^{j e t}$ as a function of rapidity are sensitive to the parton distributions in the photon. These parton distributions can be extracted by comparison of the data with QCD calculations, using an iterative procedure. However, the underlying event energy, explained in section 5.1, has a large influence on the measured jet cross sections, as will be shown below. Before conclusions may be drawn on the photon structure, the underlying event has to be described by the QCD calculation (see Fig. 4), or the jet data need to be corrected for underlying event effects (e.g. [4]).

In Fig. 7 the inclusive differential jet $e p$ cross sections $d \sigma / d E_{T}^{j e t}$ and $d \sigma / d \eta^{j e t}$ are shown. The measurements were made using the jet event sample. No corrections for the underlying event energy were applied. Here the pseudo-rapidity $\eta^{\text {jet }}$ in the laboratory system $\left(\eta-\eta^{*}=\right.$ $\left.0.5 \ln \left(E_{p} / E_{\gamma}\right) \approx 2\right)$ is chosen for compatibility with previous measurements. The cross sections are for a scaled photon energy range $0.25<y<0.7$ and negative squared momentum transfer $Q^{2}<0.01 \mathrm{GeV}^{2}$. The jet cross section as a function of the corrected transverse jet energy in Fig. 7a was measured in two pseudo-rapidity intervals $-1<\eta^{\text {jet }}<1$ and $-1<\eta^{\text {jet }}<2$ (see also tables 2,3). The jet cross section as a function of pseudo-rapidity in Fig. $7 \mathrm{~b}$ was measured for three different thresholds in the jet energy, $E_{T}^{\text {jet }}>7,11,15 \mathrm{GeV}$ (see also tables $4,5,6$ ). The previous measurement of $d \sigma / d \eta^{\text {jet }}$ for jet energies above $E_{T}^{\text {jet }}>7 \mathrm{GeV}$ [2] suffered from a defect in the acceptance correction for the small angle electron detector and is superceded by this new measurement. 

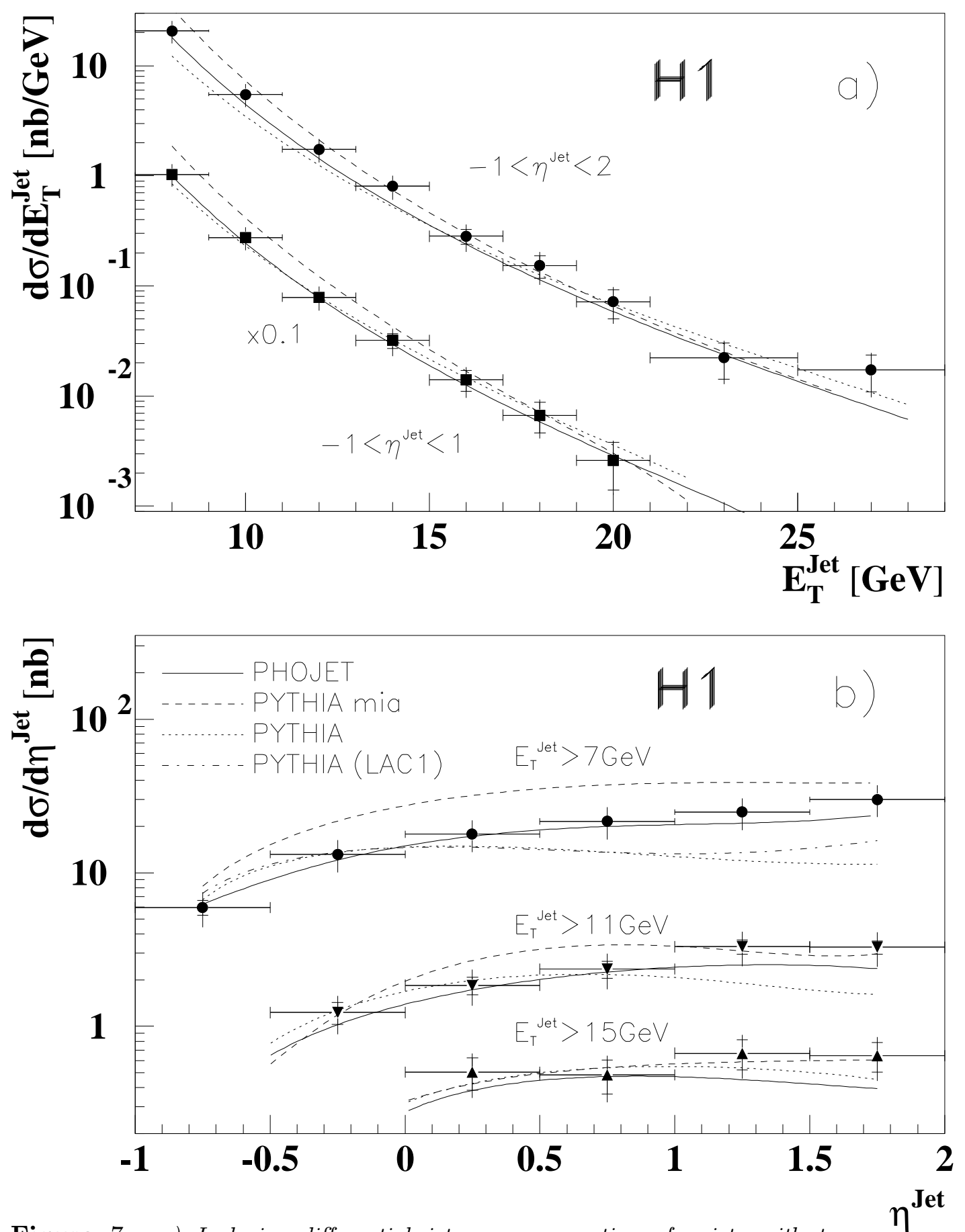

Figure 7: a) Inclusive differential jet ep cross sections for jets with transverse energy above $E_{T}^{\text {jet }}>7 \mathrm{GeV}$ summed in a cone of $R=1$. Circles are $H 1$ data integrated in the pseudo-rapidity range $-1<\eta^{\text {jet }}<2$, squares refer to the region $-1<\eta^{\text {jet }}<1$ (lowered by a factor 10). The inner error bars represent the statistical, the outer errors are the quadratic sum of statistical and systematic errors which depend on $E_{T}^{j e t}$ or $\eta^{\text {jet }}$. The uncertainty in the overall normalization amounts to $26 \%$ and is not shown in the figure. The curves show the calculations of different QCD generators with interactions of the beam remnants (full=PHOJET, dashed=PYTHIA), and without them (dotted=PYTHIA).

b) Differential jet cross section versus pseudo-rapidity for different thresholds in the jet transverse energy: circles are $H 1$ data with $E_{T}^{\text {jet }}>7 \mathrm{GeV}$, down-pointing triangles with $E_{T}^{\text {jet }}>11 \mathrm{GeV}$, and up-pointing triangles with $E_{T}^{j e t}>15 \mathrm{GeV}$. The dash-dotted curve is a calculation using PYTHIA without interactions of the beam remnants, but another parameterization of the photon structure function (see text). Other curves are described in a). 
Corrections to the energy response of the calorimeter were made as functions of the transverse energy and pseudo-rapidity of the jets. Using generator events the corrections $\epsilon$ result from the comparison of jet rates resulting from generated hadrons and jets found after a detailed simulation of detector effects. The correction function $\epsilon\left(E_{T}^{j e t}\right)$ decreases from 1.1 to 0.7 as $E_{T}^{\text {jet }}$ increases between $7<E_{T}^{\text {jet }}<30 \mathrm{GeV}$. The correction $\epsilon\left(\eta^{\text {jet }}\right)$ decreases from 1.7 to 0.7 as $\eta$ increases from -1 to 2 . The systematic uncertainty in the determination of the jet rate corrections is $10 \%$, including a small dependence on the generator model used. The hadronic energy resolution is known to the $10 \%$ level. This was found to give a $10 \%$ contribution to the systematic error. The hadronic energy scale of different calorimeter segments is known to be the same to $3 \%$. The latter results in an $15 \%$ contribution to the jet cross section error. The efficiency of the drift chamber trigger (see section 2) varies as function of $E_{T}^{\text {jet }}$ and $\eta^{\text {jet }}$ between $90 \%$ and $95 \%$, with an uncertainty of $3 \%$. In Fig. 7 the total error bars include the quadratic sum of the statistical and systematic errors mentioned so far.

Sources of systematic errors affecting the cross section normalization are as follows: 1) The dominant systematic error of $25 \%$ results from the uncertainty of $\pm 5 \%$ in the LAr energy scale. 2) The error in the luminosity measurement, which includes the uncertainty in the electron trigger efficiency, is 5\%. These errors have been added in quadrature and give a $26 \%$ overall systematic error in addition to the errors shown in Fig. 7.

The fit to a power law $\left(E_{T}^{j e t}\right)^{-n}$ of the jet cross section as a function of transverse energy in Fig. 7a gives $n=6.1 \pm 0.5$ for the pseudo-rapidity interval $-1<\eta^{j e t}<2$, where the error reflect the statistical and systematic uncertainties of the fit. The measured power $n$ is compatible with previous results on jet production in $\gamma p$ interactions $[2,3]$. This result compares also well with jet cross sections measured in $\bar{p} p$ collisions at the same cms energy $\sqrt{s_{\bar{p} p}}=200 \mathrm{GeV}$ [39] where the same fit function, applied to the same $E_{T}^{\text {jet }}$ range, results in the power $n=5.8$. Within the errors no differences between $\gamma p$ and $\bar{p} p$ are found in this kinematic range.

In Fig. 7 the calculations of the PYTHIA generator without multiple interactions (dotted curves) are compared with the measured jet cross sections. This model, and also HERWIG (not shown), show clear deficiencies in describing the cross sections of jet production at large rapidities and small transverse energies of the jets. This kinematic region corresponds to events where the momentum fraction of the parton from the photon is small $x_{\gamma} \approx 0.1$.

The PYTHIA calculation with multiple interactions provides a fair description of the shape of the measured pseudo-rapidity cross section, but gives a too large cross section in the region of small $E_{T}^{\text {jet }}>7 \mathrm{GeV}$ (dashed curves). The full curves represent the calculation of the PHOJET generator which gives a good description of both the shape and the measured rates. The results of the calculations including additional event energy - PHOJET and PYTHIA differ in absolute numbers in the small $E_{T}^{j e t}$ region despite the use of the same QCD matrix elements and structure functions. However, the calculations include different modeling of 1) the beam remnant interactions ((semi- ) hard interactions in PYTHIA versus soft and (semi- ) hard interactions in PHOJET), 2) the transition to the non-perturbative soft scattering region (unitarization concept), and 3) the parton radiation effects (hard initial state parton radiation in PYTHIA, but not in PHOJET). The comparison of these two models suggests that inferences on the parton content in the photon from the measured jet cross sections have an uncertainty of about a factor of 2 in the low $x_{\gamma}$ region.

The dash-dotted line in Fig. 7b indicates a PYTHIA calculation without multiple interactions using a different parameterization of the photon structure (LAC1 [37]) which has larger gluon content at small $x_{\gamma}$ than the parameterization used for the other calculations in this paper (GRV-LO [15]). This calculation also does not describe the measured energy density (Fig. 4) and the jet cross section measurement (Fig. 7). The same PYTHIA calculation with the LAC1 set including multiple interactions gives, after re-adjusting of the transverse momentum cut-off 
to $p_{t}^{\text {mia }} \geq 2 \mathrm{GeV}$ (compare section 5.1), a jet cross section which is between $1.5<\eta^{\text {jet }}<2$ and above $E_{T}^{\text {jet }}>7 \mathrm{GeV}$ a factor of two higher than the calculation with the GRV-LO parton distribution function in the photon, and factor 2.5 larger than the data.

The distribution of the energy density outside the jets in the central rapidity region of the $\gamma p$ collision $-1 \leq \eta^{*} \leq 1$, i.e. $1 \leq \eta \leq 3$, was used to correct the jet cross section as a function of pseudo-rapidity above $\eta^{\text {jet }}>1$ for effects of the underlying event: the difference between the transverse energy density in the data and the PYTHIA calculation without multiple interactions (Fig. 4: dotted histogram) indicates the event energy outside of jets which is produced beyond the hard parton scattering process, its parton showers and their fragmentation effects. Using the information on the true parton momentum $x_{\gamma}$ in generated events the pseudo-rapidity of the jet is correlated with $x_{\gamma}^{\text {jets }}$. With the assumption that the calculations of the PYTHIA and HERWIG generators represent a good approximation to the energy density outside the jets arising from the hard parton scattering process, the transverse jet energies are lowered by the energy difference between the data and the calculation without multiple interactions. The corrections lower the cross section for jets between $1<\eta^{\text {jet }}<2$ above $E_{T}^{\text {jet }}>7 \mathrm{GeV}$ by $40 \%$, $E_{T}^{\text {jet }}>11 \mathrm{GeV}$ by $30 \%, E_{T}^{\text {jet }}>15 \mathrm{GeV}$ by $15 \%$, and demonstrate the strong influence of the underlying event energy on the jet cross sections (see also table 7 ).

\section{Summary: Properties of jets and jet cross sections}

The width of jets in photoproduction events is observed to decrease with increasing jet energy. Comparisons of jet width, measured in $\gamma p$ and $\bar{p} p$ scattering, show that these jets are of a common nature.

Jet cross sections are measured as a function of the jet transverse energy and pseudo-rapidity. The shapes of the latter measurements are better described by LO QCD calculations which include, in addition to the matrix elements of the parton scattering processes and parton showers, interactions of the beam remnants, than by calculations without such additional interactions. The strong influence of the underlying event energy on the measured jet cross sections is demonstrated. This gives rise to uncertainties in conclusions concerning the parton scattering processes.

Table 1: Measured differential ep cross-section $d \sigma / d E_{T}$ integrated over $-1.0<\eta<2.5$ in the kinematical range defined by $Q^{2}<0.01 \mathrm{GeV}^{2}$ and $0.3 \leq y \leq 0.7$.

\begin{tabular}{|l||c|c|c|}
\hline$E_{T}[\mathrm{GeV}]$ & $d \sigma / d E_{T}[\mathrm{nb} / \mathrm{GeV}]$ & stat. error & syst. error \\
\hline \hline $20-22$ & 8.1 & 0.30 & 1.7 \\
\hline $22-24$ & 5.7 & 0.27 & 1.3 \\
\hline $24-26$ & 3.6 & 0.19 & 0.94 \\
\hline $26-28$ & 2.3 & 0.17 & 0.65 \\
\hline $28-30$ & 1.4 & 0.12 & 0.44 \\
\hline $30-32$ & 1.0 & 0.10 & 0.33 \\
\hline $32-34$ & 0.80 & 0.10 & 0.28 \\
\hline $34-36$ & 0.55 & 0.096 & 0.21 \\
\hline $36-38$ & 0.37 & 0.065 & 0.16 \\
\hline $38-41$ & 0.26 & 0.038 & 0.11 \\
\hline $41-44$ & 0.068 & 0.016 & 0.032 \\
\hline $44-50$ & 0.053 & 0.020 & 0.028 \\
\hline
\end{tabular}


Table 2: Measured differential ep cross-section $d \sigma / d E_{T}^{\text {jet }}$ for inclusive jet production integrated over $-1.0 \leq \eta^{\text {jet }} \leq 2.0$ in the kinematical range defined by $Q^{2}<0.01 \mathrm{GeV}^{2}$ and $0.25<y<0.7$.

\begin{tabular}{|c||c|c|c|c|}
\hline$E_{T}^{\text {jet }}[\mathrm{GeV}]$ & $d \sigma / d E_{T}^{\text {jet }}[\mathrm{nb} / \mathrm{GeV}]$ & stat.error & syst.error & syst.overall error \\
\hline \hline $7.0-9.0$ & 20.8 & 0.56 & 4.4 & 5.4 \\
\hline $9.0-11.0$ & 5.5 & 0.26 & 1.2 & 1.4 \\
\hline $11.0-13.0$ & 1.74 & 0.12 & 0.37 & 0.45 \\
\hline $13.0-15.0$ & 0.803 & 0.079 & 0.17 & 0.21 \\
\hline $15.0-17.0$ & 0.283 & 0.044 & 0.059 & 0.074 \\
\hline $17.0-19.0$ & 0.153 & 0.036 & 0.032 & 0.040 \\
\hline $19.0-21.0$ & 0.0715 & 0.021 & 0.015 & 0.019 \\
\hline $21.0-25.0$ & 0.0223 & 0.0081 & 0.0047 & 0.0058 \\
\hline $25.0-29.0$ & 0.0172 & 0.0063 & 0.0036 & 0.0045 \\
\hline
\end{tabular}

Table 3: Measured differential ep cross-section $d \sigma / d E_{T}^{\text {jet }}$ for inclusive jet production integrated over $-1.0 \leq \eta^{\text {jet }} \leq 1.0$ in the kinematical range defined by $Q^{2}<0.01 \mathrm{GeV}^{2}$ and $0.25<y<0.7$.

\begin{tabular}{|c||c|c|c|c|}
\hline$E_{T}^{\text {jet }}[\mathrm{GeV}]$ & $d \sigma / d E_{T}^{\text {jet }}[\mathrm{nb} / \mathrm{GeV}]$ & stat.error & syst.error & syst.overall error \\
\hline \hline $7.0-9.0$ & 10.3 & 0.39 & 2.2 & 2.7 \\
\hline $9.0-11.0$ & 2.76 & 0.19 & 0.58 & 0.72 \\
\hline $11.0-13.0$ & 0.79 & 0.08 & 0.17 & 0.20 \\
\hline $13.0-15.0$ & 0.322 & 0.049 & 0.067 & 0.084 \\
\hline $15.0-17.0$ & 0.141 & 0.030 & 0.030 & 0.037 \\
\hline $17.0-19.0$ & 0.0672 & 0.021 & 0.014 & 0.017 \\
\hline $19.0-21.0$ & 0.0263 & 0.012 & 0.0055 & 0.0068 \\
\hline
\end{tabular}

Table 4: Measured differential ep cross-section $d \sigma / d \eta^{\text {jet }}$ for inclusive jet production integrated over $E_{T}^{j e t}>7.0 \mathrm{GeV}$ in the kinematical range defined by $Q^{2}<0.01 \mathrm{GeV}^{2}$ and $0.25<y<0.7$.

\begin{tabular}{|c||c|c|c|c|}
\hline \multicolumn{1}{|c||}{$\eta^{\text {jet }}$} & $d \sigma / d \eta^{\text {jet }}[\mathrm{nb}]$ & stat.error & syst.error & syst.overall error \\
\hline \hline$-1.0--0.5$ & 5.9 & 0.64 & 1.3 & 1.5 \\
\hline$-0.5-0.0$ & 13.2 & 0.86 & 2.8 & 3.4 \\
\hline $0.0-0.5$ & 17.8 & 1.1 & 3.7 & 4.6 \\
\hline $0.5-1.0$ & 21.6 & 1.1 & 4.5 & 5.6 \\
\hline $1.0-1.5$ & 24.8 & 1.2 & 5.2 & 6.5 \\
\hline $1.5-2.0$ & 30.1 & 1.3 & 6.3 & 7.8 \\
\hline
\end{tabular}


Table 5: Measured differential ep cross-section $d \sigma / d \eta^{\text {jet }}$ for inclusive jet production integrated over $E_{T}^{j e t}>11.0 \mathrm{GeV}$ in the kinematical range defined by $Q^{2}<0.01 \mathrm{GeV}^{2}$ and $0.25<y<0.7$.

\begin{tabular}{|c||c|c|c|c|}
\hline \multicolumn{1}{|c||}{$\eta^{\text {jet }}$} & $d \sigma / d \eta^{\text {jet }}[\mathrm{nb}]$ & stat.error & syst.error & syst.overall error \\
\hline \hline$-0.5-0.0$ & 1.23 & 0.20 & 0.26 & 0.32 \\
\hline $0.0-0.5$ & 1.85 & 0.24 & 0.39 & 0.48 \\
\hline $0.5-1.0$ & 2.36 & 0.30 & 0.50 & 0.61 \\
\hline $1.0-1.5$ & 3.30 & 0.35 & 0.69 & 0.86 \\
\hline $1.5-2.0$ & 3.27 & 0.33 & 0.69 & 0.85 \\
\hline
\end{tabular}

Table 6: Measured differential ep cross-section $d \sigma / d \eta^{\text {jet }}$ for inclusive jet production integrated over $E_{T}^{\text {jet }}>15.0 \mathrm{GeV}$ in the kinematical range defined by $Q^{2}<0.01 \mathrm{GeV}^{2}$ and $0.25<y<0.7$.

\begin{tabular}{|c||c|c|c|c|}
\hline$\eta^{\text {jet }}$ & $d \sigma / d \eta^{\text {jet }}[\mathrm{nb}]$ & stat.error & syst.error & syst.overall error \\
\hline \hline $0.0-0.5$ & 0.50 & 0.12 & 0.11 & 0.13 \\
\hline $0.5-1.0$ & 0.48 & 0.12 & 0.10 & 0.13 \\
\hline $1.0-1.5$ & 0.67 & 0.15 & 0.14 & 0.17 \\
\hline $1.5-2.0$ & 0.65 & 0.14 & 0.14 & 0.17 \\
\hline
\end{tabular}

Table 7: The measured inclusive differential jet ep cross-section $d \sigma / d \eta^{j e t}$ after a subtraction of that part of the underlying event energy which is found above the calculated energy of the PYTHIA QCD generator without interactions of the beam remnants. The error bars reflect the statistical and systematic errors, added in quadrature. The kinematical range is defined by $Q^{2}<0.01 \mathrm{GeV}^{2}$ and $0.25<y<0.7$.

\begin{tabular}{|c||c|c|}
\hline $\begin{array}{c}E_{t}^{\text {jet }} \text { threshold } \\
{[\mathrm{GeV}]}\end{array}$ & $\begin{array}{c}d \sigma / d \eta^{j e t}[\mathrm{nb}] \\
\text { for } 1<\eta^{\text {jet }}<1.5\end{array}$ & $\begin{array}{c}d \sigma / d \eta^{j e t}[\mathrm{nb}] \\
\text { for } 1.5<\eta^{\text {jet }}<2\end{array}$ \\
\hline \hline 7 & $14.1 \pm 6.9$ & $17.8 \pm 8.7$ \\
\hline 11 & $2.2 \pm 1.0$ & $2.2 \pm 0.9$ \\
\hline 15 & $0.54 \pm 0.25$ & $0.52 \pm 0.24$ \\
\hline
\end{tabular}




\section{Conclusions}

In this paper detailed studies of high $E_{T}$ photoproduction events with center-of-mass energies around $\sqrt{s_{\gamma p}}=200 \mathrm{GeV}$ were presented. The selected events have large transverse energy $E_{T}>20 \mathrm{GeV}$ in the hadronic final state or exhibit the production of jets with transverse energy above $E_{T}^{\text {jet }}>7 \mathrm{GeV}$ summed in a cone of size $R=1$.

1.) The differential cross section as a function of transverse energy was measured between $20 \leq E_{T} \leq 50 \mathrm{GeV}$ in the pseudo-rapidity range $-2.5 \leq \eta^{*} \leq 1$ (Fig. 2a, Tab. 1). The shape is equally well described by a power law $E_{T}^{-n}$ with $n=5.9 \pm 0.1$, and by an exponential function $e^{-\lambda E_{T}}$ with $\lambda=0.21 \pm 0.01$.

2.) The transverse energy flow as a function of pseudo-rapidity is distinct from being flat and increases towards the origin of the $\gamma p$ center-of-mass system (Fig. 2b).

3.) Both the multi-jet rate and the transverse energy contained in jets increase with the total transverse energy (Fig. 3).

QCD calculations which include, in addition to the hard parton scattering, interactions between the beam remnants, provide better descriptions of these transverse energy distributions than models without such additional interactions.

4.) The transverse energy density in the central region of the $\gamma p$ collision outside jets in resolved photon interactions reaches $1 \mathrm{GeV} / \mathrm{rad}$ per unit area in pseudo-rapidity and $\varphi$ space and is far higher than the energy density observed in direct photon processes, $0.3 \mathrm{GeV} / \mathrm{rad}$ (Fig. 4).

5.) The transverse energy depositions show short range correlations and long range anticorrelations relative to the transverse energy measured at the origin of the $\gamma p$ center-ofmass system (Fig. 5).

QCD models without interactions of the beam remnants provide too small underlying event energy and show too strong energy correlations. In QCD models with beam remnant interactions the additional energy from the secondary interaction is essentially uncorrelated with the primary hard parton interaction. Such models not only provide the large underlying transverse energy density observed in the data, but also reproduce the energy correlations measured in the data. This supports the models including interactions between the beam remnants, although it is not inconceivable that a more complete QCD calculation of the primary scattering process may reproduce the same effects.

6.) The jets observed in $\gamma p$ collisions become denser around the jet axis with increasing jet energy (Fig. 6b).

7.) The jet cross section as a function of jet transverse energy decreases as $\left(E_{T}^{j e t}\right)^{-n}$ with $n=6.1 \pm 0.5$ between $7<E_{T}^{\text {jet }}<29 \mathrm{GeV}$ in the pseudo-rapidity range $-1<\eta^{\text {jet }}<2$ (Fig. 7a, Tab. 2, 3).

8.) The jet cross section was measured as a function of pseudo-rapidity (Fig. 7b, Tab. 4, 5, 6). The strong influence of the treatment of the underlying event energy on the cross section was demonstrated (Tab. 7). 
In order to draw conclusions on the parton scattering process, QCD jet calculations have to be compared not only to the jet cross sections (items 7,8 ), but also at least to the energy density of the underlying event (item 4).

Comparisons of the $\gamma p$ results $1,2,4,6,7$ with distributions from $\bar{p} p$ collisions show that not only are the jets of similar shape, but also the features of resolved $\gamma p$ interactions are similar to those of $\bar{p} p$ scattering. Furthermore, the analysis of direct and resolved photon interactions enabled, for the first time, the study of the underlying event energy depending on the energy available to the photon remnant, revealing new information on the multi-parton dynamics.

\section{Acknowledgments}

We are indebted to J.Chyla, R.Engel and T.Sjöstrand for help concerning the Monte Carlo event generators used in this analysis. We are grateful to the HERA machine group whose outstanding efforts made this experiment possible. We appreciate the immense effort of the engineers and technicians who constructed and maintain the detector. We thank the funding agencies for their financial support of the experiment. We wish to thank the DESY directorate for the hospitality extended to the non-DESY members of the collaboration.

\section{References}

[1] W.J. Stirling, "Proceedings of the HERA Workshop", ed. R.D. Peccei, Hamburg 1987 and references therein

[2] H1 Collaboration, I. Abt et al., Phys. Lett. B314 (1993) 436

[3] ZEUS Collaboration, M. Derrick et al., Phys. Lett. B342 (1995) 417

[4] H1 Collaboration, T. Ahmed et al., Nucl. Phys. B445 (1995) 195

[5] G. Knies, J. Phys. G, Nucl. Part. Phys. 19 (1993) 1523

[6] S.D. Ellis, Proceedings "QCD and High Energy Hadronic Interactions", 28th Rencontre de Moriond, Ed. J. Tran Thanh Van, Editions Frontieres (1993) 235

[7] T. Sjöstrand and M. van Zijl, Phys. Rev. D36 (1987) 2019

[8] G. Schuler and T. Sjöstrand, Nucl. Phys. B407 (1993) 539

[9] H1 Collaboration, I. Abt et al., DESY 93-103 (1993)

[10] H1 Calorimeter Group, B. Andrieu et al., Nucl. Instr. and Meth. A336 (1993) 460

[11] H1 Calorimeter Group, B. Andrieu et al., Nucl. Instr. and Meth. A350 (1994) 57

[12] H1 Calorimeter Group, B. Andrieu et al., Nucl. Instr. and Meth. A336 (1993) 499

[13] J.E. Huth et al., FERMILAB-CONF-90/249-E (1990)

[14] M. Glück, E. Reya and A. Vogt, Z. Phys. C53 (1992) 127

[15] M. Glück, E. Reya and A. Vogt, Phys. Rev. D46 (1992) 1973

[16] T. Sjöstrand, CERN-TH-6488 (1992), Comput. Phys. Commun. 82 (1994) 74

[17] T. Sjöstrand and M. Bengtsson, Comput. Phys. Commun. 43 (1987) 367 
[18] AFS Collaboration, T. Åkesson et al., Z. Phys. C34 (1987) 163

[19] UA2 Collaboration, J. Alitti et al., Phys. Lett. B268 (1991) 145

[20] CDF Collaboration, F. Abe et al., Phys. Rev. D47 (1993) 4857

[21] R. Engel, Z. Phys. C66 (1995) 203

[22] A. Capella et al., Phys. Rep. 236 (1994) 227

[23] P. Aurenche et al., Phys. Rev. D45 (1992) 92

F.W. Bopp et al., Comp. Phys. Commun. 83 (1994) 107

[24] A. Capella et al., Phys. Rev. Lett. 58 (1987) 2015

[25] R. Engel, F.W. Bopp, D. Pertermann and J. Ranft, Phys. Rev. D46 (1992) 5192

F.W. Bopp, R. Engel, D. Pertermann and J. Ranft, Phys. Rev. D49 (1994) 3236

[26] G. Marchesini et al., Comput. Phys. Commun. 67 (1992) 465

[27] G. Marchesini and B.R. Webber, Nucl. Phys. B310 (1988) 461

G. Marchesini and B.R. Webber, Nucl. Phys. B238 (1984) 1

[28] B.R. Webber, Nucl. Phys. B238 (1984) 492

G. Marchesini and B.R. Webber, Phys. Rev. D38 (1988) 3419

[29] J.M. Butterworth et al., CERN-TH-95-83 (1995)

[30] ZEUS Collaboration, M. Derrick et al., Phys. Lett. B297 (1992) 404

[31] AFS Collaboration, T. Åkesson et al., Phys. Lett. B128 (1983) 354

[32] P. Capiluppi et al., Nucl. Phys. B70 (1974) 1

BS Collaboration, B. Alper et al., Nucl. Phys. B100 (1975) 237

[33] UA1 Collaboration, C. Albajar et al., Z. Phys. C36 (1987) 33

[34] CDF Collaboration, F. Abe et al., Phys. Rev. D45 (1992) 2249

[35] S.D. Ellis, J. Phys. G, Nucl. Part. Phys. 17 (1991) 1552

[36] H1 Collaboration, S. Aid et al., DESY 95-156 (1995)

[37] H. Abramowicz, K. Charchula and A. Levy, Phys. Lett. B269 (1991) 458

[38] G. Kramer and S.G. Salesch, Phys. Lett. B317 (1993) 218

S.G. Salesch, DESY 93-196 (1993)

G. Kramer and S.G. Salesch, Phys. Lett. B333 (1994) 519

[39] UA1 Collaboration, C. Albajar et al., Nucl. Phys. B309 (1988) 405

[40] UA1 Collaboration, G. Arnison et al., Phys. Lett. B132 (1983) 214 